\title{
Numerical Methods for Controlled Hamilton-Jacobi-Bellman PDEs in Finance
}

\author{
P.A. Forsyth* $\quad$ G. Labahn ${ }^{\dagger}$
}

October 12, 2007

\begin{abstract}
Many nonlinear option pricing problems can be formulated as optimal control problems, leading to Hamilton-Jacobi-Bellman (HJB) or Hamilton-Jacobi-Bellman-Isaacs (HJBI) equations. We show that such formulations are very convenient for developing monotone discretization methods which ensure convergence to the financially relevant solution, which in this case is the viscosity solution. In addition, for the HJB type equations, we can guarantee convergence of a Newton-type (Policy) iteration scheme for the nonlinear discretized algebraic equations. However, in some cases, the Newton-type iteration cannot be guaranteed to converge (for example, the HJBI case), or can be very costly (for example for jump processes). In this case, we can use a piecewise constant control approximation. While we use a very general approach, we also include numerical examples for the specific interesting case of option pricing with unequal borrowing/lending costs and stock borrowing fees.
\end{abstract}

Keywords: Option pricing, stochastic control, nonlinear HJB PDE

\section{Introduction}

There are a number of financial models which result in nonlinear partial differential equations (PDEs). Examples where such nonlinear PDEs arise include transaction cost/uncertain volatility models [28, 4, 38], passport options [3, 43], unequal borrowing/lending costs [13], large investor effects [2], risk control in reinsurance [32], pricing options and insurance in incomplete markets using an instantaneous Sharpe ratio [51,31, 11], and optimal consumption [12, 15]. A recent survey article on the theoretical aspects of this topic is given in [35].

In many of these cases, the financial pricing problems can also be naturally posed as optimal control problems, leading to nonlinear Hamilton-Jacobi-Bellman (HJB) PDEs, partial integro differential equations (PIDEs) or Hamilton-Jacobi-Bellman-Isaacs (HJBI) equations.

\footnotetext{
${ }^{*}$ David R. Cheriton School of Computer Science, University of Waterloo, Waterloo ON, Canada N2L 3G1 e-mail: paforsyt@uwaterloo.ca

${ }^{\dagger}$ David R. Cheriton School of Computer Science, University of Waterloo, Waterloo ON, Canada N2L 3G1 glabahn@uwaterloo.ca
} 
A common approach found in the literature is to analytically determine the optimal control, and then substitute this control back into the PDE. Unfortunately, this method leads to PDEs which are highly nonlinear and where it is extremely difficult to design numerical schemes which are guaranteed to converge.

In this paper we consider the discrete optimal control problem directly. Our objective is to provide a general procedure for numerical solution of single factor optimal control problems in option pricing. We focus on discretization methods which are unconditionally stable, and for which convergence to the financially relevant solution is guaranteed. We place particular emphasis on the interaction of the discretization technique with the method used to solve the nonlinear discretized algebraic equations. Along the way we look at two specific examples which are interesting in their own right: unequal borrowing/lending rates and stock borrowing fees.

There are many technical issues that need to be addressed when solving optimal control problems directly. For example, since we have nonlinear PDEs, the solutions are not necessarily unique. For our problems we need to ensure that our discretization methods converge to the financially relevant solution, which in this case is the viscosity solution [18]. As demonstrated in [38], seemingly reasonable discretization methods can converge to non-viscosity solutions. We show that an optimal control formulation is in fact quite convenient for verifying monotonicity, $l_{\infty}$ stability and consistency of our discrete schemes. Using the basic results of $[10,5]$, this ensures that our numerical solutions convergence to the viscosity solution.

In terms of existing solution methods, there are two basic threads of literature concerning controlled HJB equations. One classic approach is based on a Markov chain approximation (see for example [27]). In financial terms, this approach is similar to the usual binomial lattice, which is equivalent to an explicit finite difference method. However, these methods are well-known to suffer from timestep limitations due to stability considerations.

A more recent approach is based on numerical methods which ensure convergence to the viscosity solution of the HJB equation. Unconditionally monotone implicit methods are described in [8]. This leads to a nonlinear set of discretized equations which must be solved at each timestep. It is common in the PDE literature [8] to suggest relaxation type methods for solution of the nonlinear algebraic equations at each timestep. However convergence of relaxation methods can be very slow for fine grids. On the other hand if we require a monotone scheme, then the discrete equations can be related to the discrete equations which occur in infinite horizon controlled Markov chains. If we solve a discrete version of the control problem, then in some cases we can obtain guarantees on the convergence of Newton-type (Policy) iteration schemes.

Nevertheless, there are cases where the nonlinear discrete equations are quite difficult to solve, or the convergence of the iteration is very slow. A case in point, which we will not pursue in this paper, would be the PIDE case. It may be quite difficult to solve a local control problem with a control parameter in the integral operator. An alternative possibility, is to approximate the action of the control as piecewise constant in time [25]. A simple case of this which is commonplace in finance is the approximation of an American option by a Bermudan option, with exercise at the end of each timestep. We use this same idea for other types of controls. This gives a method which has no timestep limitations due to stability, and does not require the solution of any nonlinear iterations at each timestep. In this case the controls must be discretized, and an additional PDE must be solved (at each timestep) for each discrete control. As such, this approach reduces a single 
complex nonlinear problem to a set of linear problems, with a nonlinear updating rule at the end of each timestep.

The main results of this paper are summarized as follows:

- We show that many nonlinear option pricing problems can be posed as optimal control problems, in particular unequal borrowing/lending rates and stock borrowing fees.

- If the control is handled implicitly, then the control formulation allows us to easily check the conditions required to ensure convergence of the discretization to the viscosity solution.

- The control formulation leads to natural Newton-like iteration schemes for the nonlinear algebraic equations, which arise from an implicit treatment of the control.

- The control problem can also be reformulated as piecewise constant (in time) to avoid solving nonlinear algebraic equations, at the expense of solving a number of linear problems at each timestep.

- A combination of the implicit control and piecewise constant control can be used to obtain robust and efficient methods.

We include numerical examples illustrating these ideas for a model with unequal borrowing/lending rates and stock borrowing fees. We remark that, while it is standard in the PDE literature to use a combination of forward and backward differencing to ensure monotonicity (and hence implying that the error in the space-like direction is only first order), in practical financial applications, it is usually possible to use central differencing at most nodes, and still obtain a monotone scheme. Our numerical examples illustrate that our schemes effectively have second order convergence in most cases.

\section{Preliminaries}

Let $V(S, t)$ be the value of a contingent claim written on asset $S$ which follows the stochastic process

$$
d S=\mu S d t+\sigma S d Z
$$

where $\mu$ is the drift rate, $\sigma$ is volatility, and $d Z$ is the increment of a Wiener process. There are a number of financial situations where the value of a contingent claim is determined by solving an optimal control problem.

Consider for example, the uncertain volatility model developed in $[4,30]$. This provides a pricing mechanism for cases where volatility is uncertain, but lies within a band, $\sigma \in\left[\sigma_{\min }, \sigma_{\max }\right]$. In this case, the PDE which is used to determine the value of a contingent claim is determined by the two extremal volatilities. For a short position the optimal control problem is given by

$$
V_{t}+\sup _{Q \in \hat{Q}}\left\{\frac{q_{1}^{2} S^{2}}{2} V_{S S}+S V_{S}-r V\right\}=0
$$


where $Q=\left(q_{1}\right)$ and $\hat{Q}=\left(\left\{\sigma_{\min }, \sigma_{\max }\right\}\right)$ and $r$ is the borrowing/lending rate. Replacing the sup by an inf gives the corresponding pricing equation for a long position. A PDE of precisely the same form is obtained for the Leland model of transaction costs [28].

A second example of an optimal control problem is the passport option on a trading account $[3,43]$. In this case the holder of a passport option is entitled to go long or short an underlying asset with value $S$. At the expiry of the contract, the option holder can receive the accumulated gain on the account $W$ or walk away if $W<0$. The Black-Scholes analysis involves construction of a worst case hedging portfolio which gives an optimal control problem. If we set $V=S U$, where $V(S, t)$ is the value of the short position contract, then the fair market price for $U$ is given by

$$
U_{t}+\sup _{Q \in \hat{Q}}\left\{+\frac{\sigma^{2} S^{2}}{2}\left(x-q_{1}\right)^{2} U_{x x}+\left(\left(r-\gamma-r_{c}\right) q_{1}-\left(r-\gamma-r_{t}\right) x\right) U_{x}-\gamma U\right\}=0,
$$

with $Q=\left(q_{1}\right)$ and $\hat{Q}=([-1,1])$. Here $\gamma$ is the dividend rate, $r_{c}$ is the cost of carry, $r_{t}$ is the interest rate on the trading account and $x=\frac{W}{S}$ with $-\infty<x<\infty$. We note that in this case the control ranges over a continuous interval. However, when the payoff is convex then the controls become $\hat{Q}=(\{-1,+1\})$.

A third, more recent, example of an optimal control problem occurs in pricing certain insurance contracts in incomplete markets. One possible method for pricing such contracts is based on an instantaneous Sharpe ratio [31, 11]. In [51], an example is discussed for the case of hedging with an imperfectly correlated asset. Suppose the asset underlying the contract follows the process (2.1). However, it is not possible to trade in $S$, rather only in the asset $S^{\prime}$, which follows

$$
d S^{\prime}=\mu^{\prime} S^{\prime} d t+\sigma^{\prime} S^{\prime} d Z^{\prime} \quad \text { with } \quad d Z d Z^{\prime}=\rho d t
$$

For a short position, the pricing PDE is then

$$
V_{t}+\sup _{Q \in \hat{Q}}\left\{\left(r^{\prime}+q_{1} \lambda \sigma \sqrt{1-\rho^{2}}\right) S V_{S}+\frac{\sigma^{2} S^{2}}{2} V_{S S}-r V\right\}=0
$$

where $Q=\left(q_{1}\right)$ and $\hat{Q}=(\{-1,+1\})$. Here $\lambda$ is the instantaneous Sharpe ratio and

$$
r^{\prime}=\mu-\left(\mu^{\prime}-r\right) \frac{\sigma \rho}{\sigma^{\prime}} .
$$

Replacing the sup by an inf in equation (2.5) gives the price for a long position.

While the previous three pricing PDEs are well known, we also include the following examples of optimal control problems: 


\section{Example 2.1 : Unequal Borrowing/Lending Rates}

Consider the case where the cash borrowing rate (given by $r_{b}$ ) and the lending rate (given by $r_{l}$, with $r_{b} \geq r_{l}$ ) are not necessarily equal, a model discussed, for example, in [13, 2]. The price of an option $V$ is then given by the nonlinear PDE (a brief derivation is given in Appendix A):

$$
\begin{array}{ll}
\text { Short Position: } & V_{t}+\frac{\sigma^{2} S^{2}}{2} V_{S S}+\rho\left(V-S V_{S}\right)\left(S V_{S}-V\right)=0 \\
\text { Long Position: } & V_{t}+\frac{\sigma^{2} S^{2}}{2} V_{S S}+\rho\left(S V_{S}-V\right)\left(S V_{S}-V\right)=0,
\end{array}
$$

where

$$
\rho(x)=\left\{\begin{array}{ll}
r_{l} & \text { if } x \geq 0 \\
r_{b} & \text { if } x<0
\end{array} .\right.
$$

Notice that for the short position the nonlinear problem can be posed as

$$
V_{t}+\sup _{Q \in \hat{Q}}\left\{\frac{\sigma^{2} S^{2}}{2} V_{S S}+q_{1}\left(S V_{S}-V\right)\right\}=0,
$$

where $Q=\left(q_{1}\right)$ and $\hat{Q}=\left(\left\{r_{l}, r_{b}\right\}\right)$. As before, the price for a long position option is given by replacing the sup by an inf in equation (2.9).

For a vanilla put, the bank account $B=V-S V_{S}$ is always positive. Thus $q_{1}=r_{l}$ in equation (2.9) and the equation becomes linear. Similarly, for a vanilla call the bank account $B=V-S V_{S}<0$, so that $q_{1}=r_{b}$ in equation (2.9) and the PDE again becomes linear.

At first sight, the formulation (2.9) appears to be unnecessarily complicated compared with equation (2.7). However, we will demonstrate that using an optimal stochastic control formulation results in considerable simplification of the analysis of the numerical algorithm used to solve problem (2.9). As well, the control formulation permits use of a piecewise constant policy algorithm for numerical solution of control problem (2.9), a method which is very straightforward to implement.

\section{Example 2.2 : Stock Borrowing Fees}

The borrowing/lending model in Example 2.1 can be extended to include stock borrowing fees. Such fees are effectively paid to stock lenders when a hedger shorts a stock. The stock borrowing process is described in [21]. In essence, the holder of a short position will not receive the rate $r_{l}$ on the proceeds of the short sale, but rather $r_{l}-r_{f}$, where $r_{f}$ is the stock borrowing fee. Typically, $r_{f}$ can be about 40 bps (.4\%) [52]. The nonlinear pricing PDE in the case where it is assumed that retail customers do not receive any interest on the proceeds of a short sale (i.e. $r_{f}=r_{l}$ ), is derived in [13]. The pricing equation is (for a derivation of the option pricing PDE for the general case where $r_{f} \neq r_{l}$ see Appendix A):

$$
\begin{aligned}
& \text { Short Position: } \quad V_{t}+\frac{\sigma^{2} S^{2}}{2} V_{S S}+H\left(V_{S}\right)\left[\rho\left(V-S V_{S}\right)\left(S V_{S}-V\right)\right] \\
& +H\left(-V_{S}\right)\left[\left(r_{l}-r_{f}\right) S V_{S}-\rho(V) V\right]=0 \\
& \text { Long Position: } \quad V_{t}+\frac{\sigma^{2} S^{2}}{2} V_{S S}+H\left(-V_{S}\right)\left[\rho\left(S V_{S}-V\right)\left(S V_{S}-V\right)\right] \\
& +H\left(V_{S}\right)\left[\left(r_{l}-r_{f}\right) S V_{S}-\rho(-V) V\right]=0,
\end{aligned}
$$


where $\rho(x)$ is defined in equation (2.8), and

$$
H(y)=\left\{\begin{array}{ll}
1 & \text { if } y \geq 0 \\
0 & \text { if } y<0
\end{array} .\right.
$$

For a short position we can pose our nonlinear PDE problem as the control problem

$$
V_{t}+\sup _{Q \in \hat{Q}}\left\{\frac{\sigma^{2} S^{2}}{2} V_{S S}+q_{3} q_{1}\left(S V_{S}-V\right)+\left(1-q_{3}\right)\left[\left(r_{l}-r_{f}\right) S V_{S}-q_{2} V\right]\right\}=0,
$$

where $Q=\left(q_{1}, q_{2}, q_{3}\right)$ and $\hat{Q}=\left(\left\{r_{l}, r_{b}\right\},\left\{r_{l}, r_{b}\right\},\{0,1\}\right)$. The pricing equation for the long position again only involves replacing sup by inf.

Remark 2.1 (Arbitrage Bands) The long and short prices in Examples 2.1 and 2.2 can be considered to form an arbitrage band [13]. Any market price higher than the short price or lower than the long price represents an arbitrage opportunity. No arbitrage opportunities exist for market prices which lie between the long price and the short price.

\section{Example 2.3 : American Options}

Let $V^{*}$ be the payoff of an American option. The price of an American option can be written as

$$
\min \left(-V_{t}-\left\{\frac{\sigma^{2} S^{2}}{2} V_{S S}+r S V_{S}-r V\right\}, V-V^{*}\right)=0
$$

We can also write this as a penalized control problem:

$$
V_{t}+\sup _{\mu \in\{0,1\}}\left\{\frac{\sigma^{2} S^{2}}{2} V_{S S}+r S V_{S}-r V+\mu \frac{\left(V^{*}-V\right)}{\epsilon}\right\}=0,
$$

for $\epsilon \ll 1$. For a discussion of the penalized method from an analytical point of view, see [1]. A numerical algorithm based on the penalty method is described in [22].

\section{Example 2.4 : American Options and Stock Borrowing Fees}

An interesting case arises when the Stock Borrowing model in Example 2.2 with a long position, is combined with American early exercise. This gives rise to the problem

$$
\min \left(-V_{t}-\inf _{Q \in \hat{Q}}\left\{\frac{\sigma^{2} S^{2}}{2} V_{S S}+q_{3} q_{1}\left(S V_{S}-V\right)+\left(1-q_{3}\right)\left[\left(r_{l}-r_{f}\right) S V_{S}-q_{2} V\right]\right\}, V^{*}-V\right)=0
$$

with $Q=\left(q_{1}, q_{2}, q_{3}\right), \hat{Q}=\left(\left\{r_{l}, r_{b}\right\},\left\{r_{l}, r_{b}\right\},\{0,1\}\right)$ and where $V^{*}$ is the option payoff. We can formulate this as a penalty problem

$$
V_{t}+\sup _{\mu \in\{0,1\}} \inf _{Q \in \hat{Q}}\left\{\frac{\sigma^{2} S^{2}}{2} V_{S S}+q_{3} q_{1}\left(S V_{S}-V\right)+\left(1-q_{3}\right)\left[\left(r_{l}-r_{f}\right) S V_{S}-q_{2} V\right]+\mu \frac{\left(V^{*}-V\right)}{\epsilon}\right\}=0 .
$$


Note the interesting feature of the supinf in equation (2.15). This type of problem is commonly referred to as a stochastic game. The PDE in this case is referred to as the Hamilton-JacobiBellman-Isaacs equation (HJBI). In our case, it is obvious that we can interchange the inf sup in equation (2.15), so that the Isaacs condition is satisfied, and we can expect a unique value.

All of the above examples can be described as HJB or HJBI equations. If we assume the underlying process is a jump process, we would end up with a controlled partial integro differential equation (PIDE). We will not discuss the PIDE case further in this paper, leaving this case for future work.

We will also not specifically discuss singular or impulse control problems in this paper [35]. However, singular controls can be formulated as a penalized problem [19]. It is then straightforward to use the methods described in this paper to solve the penalized formulation of a singular control. Penalty methods can also be used for impulse control.

\section{General Form for the Example Problems}

All the methods described in this paper handle problems such Examples 2.1-2.4 along with passport options, uncertain volatility models, and many other problems in finance. For concreteness, we will make use of Examples 2.1 and 2.2 from the previous section.

As is typically the case with finance problems, we solve backwards in time from the expiry date of the contract $t=T$ to $t=0$ by use of the variable $\tau=T-t$. With a slight abuse of notation, we now let $V=V(S, \tau)$ in the remainder of the paper. Set

$$
\mathcal{L}^{Q} V \equiv a(S, \tau, Q) V_{S S}+b(S, \tau, Q) V_{S}-c(S, \tau, Q) V,
$$

where the control parameter $Q$ is in general a vector, that is, $Q=\left(q_{1}, q_{2}, \ldots\right)^{\prime}$. We write our problems in the general form

$$
V_{\tau}=\sup _{Q \in \hat{Q}}\left\{\mathcal{L}^{Q} V+d(S, \tau, Q)\right\}
$$

or

$$
V_{\tau}=\inf _{Q \in \hat{Q}}\left\{\mathcal{L}^{Q} V+d(S, \tau, Q)\right\} .
$$

Here we include the $d(S, \tau, Q)$ term in equation (3.2) since it would be necessary for American options.

As an example note that the coefficients for equation (3.2) with Examples 2.1 and 2.2 are

$$
\begin{aligned}
a(S, \tau, Q) & =\frac{\sigma^{2} S^{2}}{2} \\
b(S, \tau, Q)) & = \begin{cases}S q_{1} & \text { Example 2.1 } \\
S\left(q_{3} q_{1}+\left(1-q_{3}\right)\left(r_{l}-r_{f}\right)\right) & \text { Example 2.2 }\end{cases} \\
c(S, \tau, Q) & = \begin{cases}q_{1} & \text { Example 2.1 } \\
q_{3} q_{1}+\left(1-q_{3}\right) q_{2} & \text { Example 2.2 }\end{cases} \\
d(S, \tau, Q) & =0 .
\end{aligned}
$$


In the case of American options with payoff $V^{*}$, the $a(S, \tau, Q)$ and $\left.b(S, \tau, Q)\right)$ remain the same while the other coefficients become

$$
\begin{aligned}
c(S, \tau, Q) & = \begin{cases}q_{1}+\frac{\mu}{\epsilon} & \text { Example 2.1 } \\
q_{3} q_{1}+\left(1-q_{3}\right) q_{2}+\frac{\mu}{\epsilon} & \text { Example 2.2 }\end{cases} \\
d(S, \tau, Q) & =\frac{\mu}{\epsilon} V^{*}
\end{aligned}
$$

with the new set of controls now including the addition of parameter $\mu \in\{0,1\}$.

We will assume in the following that $a(S, \tau, Q) \geq 0, c(S, \tau, Q) \geq 0$. In a financial context this corresponds to non-negative interest rates and volatilities. In general it is useful for us to explicitly separate the penalty term in equation (3.6) from the non-penalty terms. To be more specific, we assume that

$$
\begin{aligned}
c(S, \tau, Q) & =\hat{c}(S, \tau, Q)+\frac{e(S, \tau, Q)}{\epsilon} \\
d((S, \tau, Q) & =\hat{d}(S, \tau, Q)+\frac{e(S, \tau, Q) f(S, \tau)}{\epsilon} ; \epsilon \ll 1
\end{aligned}
$$

and where $\hat{c}(S, \tau, Q), e(S, \tau, Q), f(S, \tau, Q)$ are all nonnegative.

If we have an additional set of controls $P \in \hat{P}$, and define

$$
\mathcal{L}^{Q, P} V \equiv a(S, \tau, Q, P) V_{S S}+b(S, \tau, Q, P) V_{S}-c(S, \tau, Q, P) V,
$$

then, with $d=d(S, \tau, Q, P)$, the HJBI case becomes

$$
V_{\tau}=\sup _{Q \in \hat{Q}} \inf _{P \in \hat{P}}\left\{\mathcal{L}^{Q, P} V+d(S, \tau, Q, P)\right\}
$$

For brevity in the following, we will only focus on the case with the sup in equation (3.2). All the results in the following sections hold for the inf case as well. We will point out the special problems that arise when considering the HJBI case (3.8).

\subsection{Boundary Conditions}

At $\tau=0$, we set $V(S, 0)$ to the specified contract payoff. As $S \rightarrow 0$, we assume

$$
a(S, \tau, Q)=0 \quad \text { and } \quad b(S, \tau, Q) \geq 0
$$

so that equation (3.2) reduces to the problem

$$
V_{\tau}=\max _{Q \in \hat{Q}}\left\{b(0, \tau, Q) V_{S}-c(0, \tau, Q) V+d(0, \tau, Q)\right\} .
$$

In order to ensure that classical solutions exist for the uncontrolled problem, we should have the additional condition [34]

$$
\lim _{S \rightarrow 0}\left(b(S, \tau, Q)-a_{S}(S, \tau, Q)\right) \geq 0
$$


so that no boundary condition (other than equation (3.10)) is required at $S=0$. For the CIR model, the nonuniqueness of the classical solution when condition (3.11) is not satisfied is discussed in [23].

As $S \rightarrow \infty$, we normally use financial reasoning to determine the asymptotic form of the solution. A typical assumption is that $V_{S S} \simeq 0$ [50], so that

$$
V \simeq B(\tau) S+C(\tau) ; \quad S \rightarrow \infty
$$

We make the approximation that the optimal control $Q$ is independent of time and $S$ as $S \rightarrow \infty$, so that $Q$ can be determined from the payoff as $S \rightarrow \infty$. This then leads to a set of ODEs to solve for $B(\tau), C(\tau)$ [50], with $B(0), C(0)$ determined from the contract payoff. We will assume in the following that the asymptotic form

$$
V\left(S_{\max }, \tau\right)=B(\tau) S_{\max }+C(\tau)
$$

is known.

For computational purposes, we solve problem (3.2) on

$$
0 \leq \tau \leq T \quad \text { and } \quad 0 \leq S \leq S_{\max }
$$

with condition (3.10) imposed at $S=0$, and the condition (3.13) with $B, C$ known functions imposed at $S=S_{\max }$. As pointed out in [7], we can expect any errors incurred by imposing approximate boundary conditions at $S=S_{\max }$ to be small in areas of interest if $S_{\max }$ is sufficiently large.

Assumption 3.1 (Properties of the HJB and HJBI PDE.) We make the assumption that the coefficients $a, b, c, d$ are continuous functions of $(S, \tau, Q)$, with $a \geq 0$, and $c \geq 0$ and that $a, b, \hat{c}, e, \hat{d}, f$ (equation (3.6)) are bounded on $0 \leq S \leq S_{\text {max }}$. Since we restrict ourselves to a finite computational domain $0 \leq S \leq S_{\max }$, we avoid difficulties associated with coefficients that grow with $S$ as $S \rightarrow \infty$. We also assume that the set of admissible controls $\hat{Q}$ (for the HJB case) and $\hat{Q}, \hat{P}$ (for the HJBI case) are compact (i.e. a closed, bounded interval). It follows from [16, 9] that solutions to equation (3.4) along with the boundary conditions (3.10) and (3.13) satisfy the strong comparison property, in the case that the penalty terms are zero. From [1], we know that the penalized equation is also a good approximation to the viscosity solution. Comparison results for the HJBI equation (under more general conditions than discussed in this paper) are given in [29]. Consequently, in all cases, we make the assumption that the strong comparison property holds, so that a unique viscosity solution exists for equations (3.2), (3.3), and (3.8).

Remark 3.1 (Interpretation of the Strong Comparison Property) As noted in [17], in a financial context, the strong comparison property simply states that if $W(S, \tau)$ and $V(S, \tau)$ are two contingent claims, with $W(S, 0) \geq V(S, 0)$, then $W(S, \tau) \geq V(S, \tau)$ for all $\tau$. We will verify that the schemes developed in this paper satisfy a discrete version of the comparison principle. 


\section{Discretization}

In this section, we will introduce the basic discretization for the PDE in the general form (3.2), and introduce the matrix notation to be used in the remainder of the this paper. We will discuss the concept of a positive coefficient discretization, which will ensure convergence to the viscosity solution. In addition, the positive coefficient property will allow us to prove convergence of iterative schemes for solving the nonlinear discretized algebraic equations.

Define a grid $\left\{S_{0}, S_{1}, \ldots, S_{p}\right\}$ with $S_{p}=S_{\max }$, and let $V_{i}^{n}$ be a discrete approximation to $V\left(S_{i}, \tau^{n}\right)$. Let $V^{n}=\left[V_{0}^{n}, \ldots, V_{p}^{n}\right]^{\prime}$, and let $\left(\mathcal{L}_{h}^{Q} V^{n}\right)_{i}$ denote the discrete form of the differential operator (3.4) at node $\left(S_{i}, \tau^{n}\right)$. The operator (3.4) can be discretized using forward, backward or central differencing in the $S$ direction to give

$$
\left(\mathcal{L}_{h}^{Q} V^{n+1}\right)_{i}=\alpha_{i}^{n+1}(Q) V_{i-1}^{n+1}+\beta_{i}^{n+1}(Q) V_{i+1}^{n+1}-\left(\alpha_{i}^{n+1}(Q)+\beta_{i}^{n+1}(Q)+c_{i}^{n+1}(Q)\right) V_{i}^{n+1} .
$$

Here $\alpha_{i}, \beta_{i}$ are defined in Appendix C.

It is important that central, forward or backward discretizations be used to ensure that (4.3) is a positive coefficient discretization. To be more precise, this condition is

\section{Condition 4.1 Positive Coefficient Condition}

$$
\alpha_{i}^{n+1}(Q) \geq 0, \quad \beta_{i}^{n+1}(Q) \geq 0, \quad c_{i}^{n+1}(Q) \geq 0 . \quad i=0, . ., p-1 ; \forall Q \in \hat{Q} .
$$

We will assume that all models have $c_{i}^{n+1}(Q) \geq 0$. Consequently, we choose central, forward or backward differencing at each node to ensure that $\alpha_{i}^{n+1}(Q), \beta_{i}^{n+1}(Q) \geq 0$. Note that different nodes can have different discretization schemes. If we use forward and backward differencing, then the equation (C.3) guarantees a positive coefficient method. However, since this discretization is only first order correct, it is desirable to use central differencing as much as possible (and yet still obtain a positive coefficient method). This is discussed in detail in [49].

Equation (3.2) can now be discretized using fully implicit timestepping $(\theta=0)$ or CrankNicolson $(\theta=1 / 2)$ along with the discretization (4.1) to give

$$
\frac{V_{i}^{n+1}-V_{i}^{n}}{\Delta \tau}=(1-\theta) \sup _{Q^{n+1} \in \hat{Q}}\left\{\left(\mathcal{L}_{h}^{Q^{n+1}} V^{n+1}\right)_{i}+d_{i}^{n+1}\right\}+\theta \sup _{Q^{n} \in \hat{Q}}\left\{\left(\mathcal{L}_{h}^{Q^{n}} V^{n}\right)_{i}+d_{i}^{n}\right\}
$$

These discrete equations are highly nonlinear in general. We refer to methods which use an implicit timestepping method where the control is handled implicitly as an implicit control method in the following.

\subsection{Order of Approximation}

Set

$$
(\Delta S)_{\max }=\max _{i}\left(S_{i+1}-S_{i}\right) \quad \text { and } \quad(\Delta S)_{\min }=\min _{i}\left(S_{i+1}-S_{i}\right)
$$

and suppose $\phi(S, \tau)$ is a smooth test function with bounded derivatives of all orders with respect to $(S, \tau)$. If $\phi_{i}^{n}=\phi\left(S_{i}, \tau^{n}\right)$, then using Taylor series expansions (and the discretization described 
in Appendix C) verifies that

$$
\left|\left(\mathcal{L}_{h}^{Q} \phi\right)_{i}^{n}-\left(\mathcal{L}^{Q} \phi\right)_{i}^{n}\right|=O\left((\Delta S)_{\max }\right)
$$

For $\phi$ a smooth test function, using equations (4.4), (B.4), (and Taylor series expansions) also gives the order of our discretization as

$$
\begin{aligned}
\mid\left(\phi_{\tau}\right)_{i}^{n+1}- & \sup _{Q \in \hat{Q}}\left\{\mathcal{L}^{Q} \phi+d\right\}_{i}^{n+1}-\left[\frac{\phi_{i}^{n+1}-\phi_{i}^{n}}{\Delta \tau}-(1-\theta) \sup _{Q^{n+1} \in \hat{Q}}\left\{\left(\mathcal{L}_{h}^{Q^{n+1}} \phi^{n+1}\right)_{i}^{n+1}+d_{i}^{n+1}\right\}\right. \\
\leq & -\theta \sup _{Q^{n} \in \hat{Q}}\left\{\left(\phi_{\tau}\right)_{i}^{n+1}-\frac{\phi_{i}^{n+1}-\phi_{i}^{n}}{\Delta \tau} \mid\right. \\
& \left.+\sup _{Q \in \hat{Q}} \mid\left\{\mathcal{L}^{Q+1}\right)_{i}^{n+1}+d_{i}^{n}\right\} \\
= & O(\Delta \tau)+O\left((\Delta S\}_{i}^{n+1}-(1-\theta)\left\{\left(\mathcal{L}_{h}^{Q} \phi^{n+1}\right)_{i}^{n+1}+d_{i}^{n+1}\right\}-\theta\left\{\left(\mathcal{L}_{h}^{Q} \phi^{n}\right)_{i}^{n}+d_{i}^{n}\right\} \mid\right. \\
= & O(\Delta \tau)+O\left((\Delta S)_{\max }\right) .
\end{aligned}
$$

The last step follows since the coefficients of the PDE are assumed continuous functions of time.

Remark 4.1 (Second Order Error) We have expanded the Taylor series in equation (4.5) about the point $\left(S_{i}, \tau^{n+1}\right)$. If we expand about the point $\left(S_{i}, \tau^{n+1 / 2}\right)$ (where $\left.\tau^{n+1 / 2}=\left(\tau^{n+1}+\tau^{n}\right) / 2\right)$ and assume that the PDE coefficients have bounded second derivatives with respect to time, then for $\theta=1 / 2$, the time truncation error is $O\left((\Delta \tau)^{2}\right)$. As well, if we assume that the grid in the $S$ direction is slowly varying, and that central weighting is used, then the error in the $S$ direction will be $O\left((\Delta S)_{\max }^{2}\right)$. In general, of course, these assumptions may not be justified. However, in many cases in practice, we observe close to second order convergence at most nodes of interest if we use Crank-Nicolson weighting.

We require our discretization to satisfy $\alpha_{i}^{n+1}, \beta_{i}^{n+1} \geq 0$ and so require a combination of forward/backward/central differencing choices. Of course we would like to use central differencing as much as possible, rather than forward/backward differencing (which are only first order correct) (see the algorithm described in [20]). However this does imply that the discretization in Appendix $\mathrm{C}$ is formally only first order accurate in $(\Delta S)_{\max }$ due to the possibility of using forward/backward differencing at some nodes, as well as the unequally spaced grid. In practice, forward/backward differencing is usually only required at a small number of nodes, and usually the grid size is changed smoothly near regions of interest. The example computations will show near quadratic convergence as the mesh size is reduced.

From a practical standpoint, there are essentially two important cases. 


\subsection{Q Independent Discretization}

In some cases, we can preselect central, forward or backward differencing independent of the optimal control $Q_{i}^{n+1}$, which ensures that the positive coefficient condition (4.2) is satisfied. In this situation, the determination of the optimal control $Q_{i}^{n+1}$, for given $\left\{V_{i}^{n+1}, V_{i+1}^{n+1}, V_{i-1}^{n+1}\right\}$ is usually straightforward. As a result, we would expect that iterative solution of the nonlinear equations (4.3) is at least feasible.

The following method is used to preselect the discretization method at each node [51]. We process each node in turn, first testing to see if central differencing satisfies (4.2), for any $Q \in \hat{Q}$. If this is the case, then we use central differencing at this node, and proceed on to the next node. If central differencing does not ensure a positive coefficient discretization, then forward and backward differencing are tested. We remark that for the problems in Examples 2.1 and 2.2, as long as $r_{l}-r_{f} \geq 0$, then one of central or forward differencing will satisfy the positive coefficient condition, for an arbitrary choice of grid, for any $Q \in \hat{Q}$.

In some cases, $Q$ independent discretization may not be possible for an arbitrary grid, but can be achieved for small enough node spacing. Usually, the problem nodes are few in number, and located near $S \rightarrow 0$, that is, where the diffusion term is small. In this case, we can often take an arbitrary grid, and insert a relatively small number of nodes, which will guarantee that $Q$ independent discretization will satisfy (4.2). An example of this node insertion algorithm is given in [51].

\subsection{Q Dependent Discretization}

Unfortunately there are some situations where no matter how fine the grid, it may not be possible to preselect the type of discretization at each node which will ensure that the positive coefficient condition (4.2) is satisfied at each node for any $Q \in \hat{Q}$. This is the case for passport options when there are non-convex payoffs $[37,49]$. In this case, the discretization at node $i$ (central, forward or backward) will depend on $Q_{i}^{n+1}$. Of course, the optimal value of $Q_{i}^{n+1}$ will now depend on the discretization. In addition, for given $\left\{V_{i}^{n+1}, V_{i+1}^{n+1}, V_{i-1}^{n+1}\right\}$, determination of the optimal value for $Q_{i}^{n+1}$ may not be straightforward. This follows since the discretized equations are continuous functions of $Q$ if forward and backward differencing only are used for the first order terms, but the discrete equations will not, in general, be continuous functions of $Q$ if central weighting is used as much as possible. This issue is discussed in detail in [49]. In the following, we will not require that the discrete equations be a continuous function of the control, to allow for the case described in [49].

\subsection{Matrix Form of the Discrete Equations}

It will be convenient to use matrix notation for equations (4.3), coupled with boundary conditions (3.10) and (3.13).

Let the boundary conditions at $S=S_{\max }$ and time $\tau^{n}$ be given by

$$
F_{p}^{n}=B\left(\tau^{n}\right) S_{\max }+C\left(\tau^{n}\right)
$$


where $S_{p}=S_{\max }$ and $B(0), C(0)$ determined from the payoff. Set

$$
V^{n}=\left[V_{0}^{n}, V_{1}^{n}, \ldots, V_{p}^{n}\right]^{\prime} \text { and } Q=\left[Q_{0}, Q_{1}, \ldots, Q_{p}\right]^{\prime}
$$

We can write the discrete operator $\left(\mathcal{L}_{h}^{Q} V^{n}\right)_{i}$ as

$$
\begin{aligned}
\left(\mathcal{L}_{h}^{Q} V^{n}\right)_{i} & =\left[A(Q) V^{n}\right]_{i} \\
& =\left[\alpha_{i}^{n}(Q) V_{i-1}^{n}+\beta_{i}^{n}(Q) V_{i+1}^{n}-\left(\alpha_{i}^{n}(Q)+\beta_{i}^{n}(Q)+c_{i}^{n}(Q)\right) V_{i}^{n}\right] ; \quad i<p .
\end{aligned}
$$

The first and last rows of $A$ are modified as needed to handle the boundary conditions. The boundary condition at $S=0$ (equation (3.10)) is enforced by setting $\alpha_{i}=0$, and using forward differencing for the first order term at $i=0$. For notational consistency, this is consistent with the above if we define $V_{-1}^{n}=0$. Let $F^{n}=\left[0, \ldots, 0, F_{p}^{n}\right]^{\prime}$. The boundary condition at $i=p$ is enforced by setting the last row of $A$ to be identically zero. With a slight abuse of notation, we denote this last row as $\left(A^{n}(Q)\right)_{p} \equiv 0$. In the following, it will be understood that equations of type (4.8) hold only for $i<p$, with $\left(A^{n}(Q)\right)_{p} \equiv 0$.

Let $D^{n}(Q)$ be the vector with entries

$$
[D(Q)]_{i}^{n}=\left\{\begin{array}{l}
d_{i}^{n}(Q), \quad i<p \\
0, \quad i=p
\end{array}\right.
$$

Remark 4.2 (Matrix Supremum Notational Convention) In the following, we will denote

$$
\sup _{Q \in \hat{Q}}\left\{\left[A^{n+1}(Q) V^{n+1}+D^{n+1}(Q)\right]_{i}\right\}
$$

by

$$
\begin{aligned}
& A^{n+1}\left(Q^{n+1}\right) V^{n+1}+D^{n+1}\left(Q^{n+1}\right) \\
& \text { where } Q_{i}^{n+1} \in \underset{Q \in \hat{Q}}{\arg \sup }\left\{\left[A^{n+1}(Q) V^{n+1}+D^{n+1}(Q)\right]_{i}\right\} .
\end{aligned}
$$

If the local objective function is a continuous function of $Q$, then, since $\hat{Q}$ is compact, the supremum is simply the maximum value, and $Q^{n+1}$ is the point where a maximum is attained. If the local objective function is discontinuous, we interpret $A^{n+1}\left(Q^{n+1}\right)$ as the appropriate limiting value of $\left[A^{n+1}(Q)\right]_{i}$ which generates the supremum, at the limit point $Q^{n+1}$. A specific example of an algorithm for computing this limit point is given for the case of maximizing the usage of central weighting as much as possible in [49]. Note that $Q^{n+1}$ is not necessarily unique.

The discrete equations (4.3) can be written as

$$
\begin{aligned}
{\left[I-(1-\theta) \Delta \tau A^{n+1}\left(Q^{n+1}\right)\right] V^{n+1}=} & {\left[I+\theta \Delta \tau A^{n}\left(A^{n}\right)\right] V^{n}+(1-\theta) \Delta \tau D^{n+1}\left(Q^{n+1}\right) } \\
& +\theta \Delta \tau D^{n}\left(Q^{n}\right)+\left(F^{n+1}-F^{n}\right), \\
\text { where } Q_{i}^{n+1} \in & \underset{Q \in \hat{Q}}{\arg \sup }\left\{\left[A^{n+1}(Q) V^{n+1}+D^{n+1}(Q)\right]_{i}\right\} \quad i=0, \ldots, p-1 .
\end{aligned}
$$


Here the term $\left(F^{n+1}-F^{n}\right)$ enforces the boundary condition at $S=S_{p}$. Recall that Crank Nicolson $(\theta=1 / 2)$ or fully implicit $(\theta=0)$ timestepping is used.

It will be convenient to define the following

$$
(\Delta \tau)_{\max }=\max _{n}\left(\tau^{n+1}-\tau^{n}\right) \quad \text { and } \quad(\Delta \tau)_{\min }=\min _{n}\left(\tau^{n+1}-\tau^{n}\right)
$$

where we assume that there are mesh size/timestep parameters $h_{\min }, h_{\max }$ such that

$$
\begin{aligned}
(\Delta S)_{\max } & =C_{1} h_{\max }, \quad(\Delta \tau)_{\max }=C_{2} h_{\max } \\
(\Delta S)_{\min } & =C_{3} h_{\min }, \quad(\Delta \tau)_{\min }=C_{4} h_{\min }
\end{aligned}
$$

with $C_{1}, C_{2}, C_{3}, C_{4}$ positive constants independent of $h$. We can then write the discrete equations (4.3) or (4.12) at each node in the following form

$$
G_{i}^{n+1}\left(h_{\max }, V_{i}^{n+1}, V_{i+1}^{n+1}, V_{i-1}^{n+1}, V_{i}^{n}, V_{i+1}^{n}, V_{i-1}^{n}\right)=0
$$

where

$$
\begin{gathered}
G_{i}^{n+1} \equiv \frac{V_{i}^{n+1}-V_{i}^{n}}{\Delta \tau}-(1-\theta) \sup _{Q^{n+1} \in \hat{Q}}\left\{\left(A^{n+1}\left(Q^{n+1}\right) V^{n+1}+D^{n+1}\left(Q^{n+1}\right)\right)_{i}\right\} \\
-\frac{F_{i}^{n+1}-F_{i}^{n}}{\Delta \tau}-\theta \sup _{Q^{n} \in \hat{Q}}\left\{\left(A^{n}\left(Q^{n}\right) V^{n}+D^{n}\left(Q^{n}\right)\right)_{i}\right\} .
\end{gathered}
$$

To avoid longwinded notation, we shall occasionally write

$$
G_{i}^{n+1}\left(h_{\max }, V_{i}^{n+1},\left\{V_{j}^{n+1}\right\}_{j \neq i},\left\{V_{j}^{n}\right\}\right) \equiv G_{i}^{n+1}\left(h_{\max }, V_{i}^{n+1}, V_{i+1}^{n+1}, V_{i-1}^{n+1}, V_{i}^{n}, V_{i+1}^{n}, V_{i-1}^{n}\right),
$$

where $\left\{V_{j}^{n+1}\right\}_{j \neq i}$ is the set of values $V_{j}^{n+1}, j=1, \ldots, p, j \neq i$, and $\left\{V_{j}^{n}\right\}$ is the set of values $V_{j}^{n}, j=1, \ldots, p$.

\section{Convergence to the Viscosity Solution}

In [38], examples were given in which seemingly reasonable discretizations of nonlinear option pricing PDEs were unstable or converged to the incorrect solution. It is important to ensure that we can generate discretizations which are guaranteed to converge to the viscosity solution $[5,18]$. Assuming that equation (3.2) satisfies the strong comparison property [6, 9, 16], then, from [10, 5], a numerical scheme converges to the viscosity solution if the method is consistent, stable (in the $l_{\infty}$ norm) and monotone. To be precise, we define these terms.

Definition 5.1 (Stability) Discretization (4.14) is stable if

$$
\left\|V^{n+1}\right\|_{\infty} \leq C_{5}
$$

for $0 \leq n \leq N, T=N \Delta \tau$, for $(\Delta \tau)_{\min } \rightarrow 0,(\Delta S)_{\min } \rightarrow 0$, where $C_{5}$ is independent of $(\Delta \tau)_{\min },(\Delta S)_{\min }$ 
Definition 5.2 (Consistency) Scheme (4.14) is consistent if, for any smooth function $\phi$, with $\phi_{i}^{n}=\phi\left(S_{i}, \tau^{n}\right)$, we have

$$
\lim _{h_{\text {max }} \rightarrow 0}\left|\left(\phi_{\tau}-\sup _{Q \in \hat{Q}}\left\{\mathcal{L}^{Q} \phi+d\right\}\right)_{i}^{n+1}-G_{i}^{n+1}\left(h_{\max }, \phi_{i}^{n+1}, \phi_{i+1}^{n+1}, \phi_{i-1}^{n+1}, \phi_{i}^{n}, \phi_{i+1}^{n}, \phi_{i-1}^{n}\right)\right|=0 .
$$

For the general case where the operator is degenerate, a more complicated definition of consistency is required in order to handle boundary data [5]. In our case, the degeneracy occurs at $S \rightarrow 0$, and boundary condition (3.10) is simply the limit of equation (3.2) as $S \rightarrow 0$. As such this problem does not arise.

The most interesting requirement is monotonicity.

Definition 5.3 (Monotonicity) The discrete scheme (4.14) is monotone if for all $\epsilon_{j}^{l} \geq 0$ and $i$

$$
G_{i}^{n+1}\left(h_{\max }, V_{i}^{n+1},\left\{V_{j}^{n+1}+\epsilon_{j}^{n+1}\right\}_{j \neq i},\left\{V_{j}^{n}+\epsilon_{j}^{n}\right\}\right) \leq G_{i}^{n+1}\left(h_{\max }, V_{i}^{n+1},\left\{V_{j}^{n+1}\right\}_{j \neq i},\left\{V_{j}^{n}\right\}\right) .
$$

Stability and consistency are easily established.

Lemma 5.1 (Stability) If the discretization (4.14) satisfies the positive coefficient condition (4.2), and boundary conditions are imposed at $S=0$ and $S=S_{\max }$, as in equation (3.10) and (3.13), then the scheme (4.12) satisfies (for $S_{\text {max }}$ fixed, and recalling the definitions of $\hat{d}, f$ in equation (3.6))

$$
\left\|V^{n}\right\|_{\infty} \leq \max \left(\left\|V^{0}\right\|_{\infty}+C_{6}, C_{7}, C_{8}\right)
$$

where $C_{6}=T \max _{i, n}\left|\hat{d}_{i}^{n}\right|, C_{7}=\max _{i, n}\left|F_{i}^{n}\right|$, and $C_{8}=\max _{i, n} f_{i}^{n}$ provided that

$$
\Delta \tau \theta\left(\alpha_{i}^{n}+\beta_{i}^{n}+c_{i}^{n}\right) \leq 1 ; \forall i
$$

Proof. For the fully implicit case $(\theta=0)$, the discrete equations are, for $i<p$,

$$
\begin{aligned}
V_{i}^{n+1}= & V_{i}^{n}-\Delta \tau\left(\alpha_{i}^{n+1}+\beta_{i}^{n+1}+\hat{c}_{i}^{n+1}+\frac{e_{i}^{n+1}}{\epsilon}\right) V_{i}^{n+1} \\
& +\Delta \tau \alpha_{i}^{n+1} V_{i-1}^{n+1}+\Delta \tau \beta_{i}^{n+1} V_{i+1}^{n+1}+\Delta \tau \hat{d}_{i}^{n+1}+\frac{e_{i}^{n+1} \Delta \tau f_{i}^{n+1}}{\epsilon}
\end{aligned}
$$

and $V_{p}^{n+1}=F_{p}^{n+1}$ when $i=p$. To avoid notational clutter, we have suppressed the $Q$ dependence in equations (5.6). It will be understood that the coefficients are the limiting values at the optimal $Q$. From equation (5.6), we obtain

$$
\begin{aligned}
\left|V_{i}^{n+1}\right|\left(1+\Delta \tau\left(\alpha_{i}^{n+1}+\beta_{i}^{n+1}+\hat{c}_{i}^{n+1}+\frac{e_{i}^{n+1}}{\epsilon}\right)\right) \leq & \left\|V^{n}\right\|_{\infty}+\left\|V^{n+1}\right\|_{\infty} \Delta \tau\left(\alpha_{i}^{n+1}+\beta_{i}^{n+1}\right) \\
& +\frac{e_{i}^{n+1} \Delta \tau f_{i}^{n+1}}{\epsilon}+\Delta \tau\left|\hat{d}_{i}^{n+1}\right| .
\end{aligned}
$$


If $\left\|V^{n+1}\right\|_{\infty}=\left|V_{j}^{n+1}\right|, j<p$, then equation (5.7) gives

$$
\left\|V^{n+1}\right\|_{\infty}\left(1+\Delta \tau \hat{c}_{j}^{n+1}+\Delta \tau \frac{e_{j}^{n+1}}{\epsilon}\right) \leq\left\|V^{n}\right\|_{\infty}+\frac{e_{j}^{n+1} \Delta \tau f_{j}^{n+1}}{\epsilon}+\Delta \tau\left|\hat{d}_{j}^{n+1}\right|,
$$

or, letting $f_{m a x}^{n+1}=\max _{j} f_{j}^{n+1}$ and $\hat{d}_{\max }^{n+1}=\max _{j}\left|\hat{d}_{j}^{n+1}\right|$, we obtain

$$
\left\|V^{n+1}\right\|_{\infty} \leq \max \left(\left\|V^{n}\right\|_{\infty}, f_{\max }^{n+1}\right)+\Delta \tau \hat{d}_{\max }^{n+1}
$$

If $j=p$ then $\left\|V^{n+1}\right\|_{\infty}=\left|V_{p}^{n+1}\right|$ and so equation $V_{p}^{n+1}=F_{p}^{n+1}$ gives

$$
\left\|V^{n+1}\right\|_{\infty}=\left|F_{p}^{n+1}\right|
$$

Combining equations (5.9) and (5.10) gives

$$
\left\|V^{n+1}\right\|_{\infty} \leq \max \left(\left\|V^{n}\right\|_{\infty}, f_{\max }^{n+1},\left|F_{p}^{n+1}\right|\right)+\Delta \tau \hat{d}_{\max }^{n+1},
$$

which then results in equation (5.4). A similar series of steps for $\theta>0$ shows that the discretization is stable provided condition (5.5) holds.

Lemma 5.2 (Consistency) If the discrete equation coefficients are as given in Appendix $C$, then the discrete scheme (4.14) is consistent as defined in Definition 5.2.

Proof. This follows from equation (4.5).

The fact that a discretization of a control problem which satisfies the positive coefficient condition (4.2) results in a monotone scheme was noted in [8]. This result holds for both $Q$ dependent and $Q$ independent discretizations (see Sections 4.2 and 4.3). It is instructive to include a proof of this result, since it illustrates the importance of maximizing/minimizing the discretized equations.

Lemma 5.3 (Monotonicity) If the discretization (4.14) satisfies the positive coefficient condition (4.2), boundary conditions are imposed at $S=0$ and $S=S_{\max }$, as in equation (3.10) and (3.13), and the stability condition (5.5) is satisfied, then discretization (4.14) is monotone as defined in Definition 5.3.

Proof. Consider the fully implicit case $(\theta=0$ in equation (4.14)). For $i=p$, the Lemma is trivially true. For $i<p$, we write equation (4.14) out in component form

$$
\begin{aligned}
& G_{i}^{n+1}\left(h, V_{i}^{n+1}, V_{i+1}^{n+1}, V_{i-1}^{n+1}, V_{i}^{n}\right) \\
& =\frac{V_{i}^{n+1}-V_{i}^{n}}{\Delta \tau}+\inf _{Q^{n+1} \in \hat{Q}}\left\{\left(\alpha_{i}^{n+1}(Q)+\beta_{i}^{n+1}(Q)+c_{i}^{n+1}(Q)\right) V_{i}^{n+1}\right. \\
& \left.\quad-\alpha_{i}^{n+1}(Q) V_{i-1}^{n+1}-\beta_{i}^{n+1}(Q) V_{i+1}^{n+1}-d_{i}^{n+1}(Q)\right\} .
\end{aligned}
$$


For $\epsilon \geq 0$, we have

$$
\begin{aligned}
& G_{i}^{n+1}\left(h, V_{i}^{n+1}, V_{i+1}^{n+1}+\epsilon, V_{i-1}^{n+1}, V_{i}^{n}\right)-G_{i}^{n+1}\left(h, V_{i}^{n+1}, V_{i+1}^{n+1}, V_{i-1}^{n+1}, V_{i}^{n}\right) \\
& =\inf _{Q \in \hat{Q}}\left\{\left(\alpha_{i}^{n+1}(Q)+\beta_{i}^{n+1}(Q)+c_{i}^{n+1}(Q)\right) V_{i}^{n+1}-\alpha_{i}^{n+1}(Q) V_{i-1}^{n+1}-\beta_{i}^{n+1}(Q) V_{i+1}^{n+1}-\beta_{i}^{n+1}(Q) \epsilon-d_{i}^{n+1}(Q)\right\} \\
& -\inf _{Q^{*} \in \hat{Q}}\left\{\left(\alpha_{i}^{n+1}\left(Q^{*}\right)+\beta_{i}^{n+1}\left(Q^{*}\right)+c_{i}^{n+1}\left(Q^{*}\right)\right) V_{i}^{n+1}-\alpha_{i}^{n+1}\left(Q^{*}\right) V_{i-1}^{n+1}-\beta_{i}^{n+1}\left(Q^{*}\right) V_{i+1}^{n+1}-d_{i}^{n+1}\left(Q^{*}\right)\right\} \\
& \leq \sup _{Q \in \hat{Q}}\left\{-\beta_{i}^{n+1}(Q) \epsilon\right\}=-\epsilon \inf _{Q \in \hat{Q}}\left\{\beta_{i}^{n+1}(Q)\right\} \leq 0,
\end{aligned}
$$

which follows from equation (B.2) and the fact that $\beta_{i}^{n+1}(Q) \geq 0$. Similarly $(\theta=0)$,

$$
G_{i}^{n+1}\left(h, V_{i}^{n+1}, V_{i+1}^{n+1}, V_{i-1}^{n+1}+\epsilon, V_{i}^{n}\right)-G_{i}^{n+1}\left(h, V_{i}^{n+1}, V_{i+1}^{n+1}, V_{i-1}^{n+1}, V_{i}^{n}\right) \leq 0 .
$$

It is obvious from equation (5.12) that $(\theta=0)$

$$
G_{i}^{n+1}\left(h, V_{i}^{n+1}, V_{i+1}^{n+1}, V_{i-1}^{n+1}, V_{i}^{n}+\epsilon\right)-G_{i}^{n+1}\left(h, V_{i}^{n+1}, V_{i+1}^{n+1}, V_{i-1}^{n+1}, V_{i}^{n}\right) \leq 0 .
$$

Finally, for the general case with $\theta \neq 0$, a similar argument verifies that property (5.3) holds, as long as the stability condition

$$
\Delta \tau \theta\left(\alpha^{n}(Q)+\beta_{i}^{n}(Q)+c_{i}^{n}(Q)\right) \leq 1 ; \forall i, \forall Q \in \hat{Q},
$$

is satisfied.

Remark 5.1 (Extension to Other Cases) Using properties (B.3), (B.6), we can replace the sup in equation (5.13) by an inf, or a sup inf (with two control variables $Q, P$ as in equation (3.8)) and the discretization is monotone for these cases as well.

Theorem 5.1 (Convergence to the Viscosity Solution) Provided that the original HJB satisfies Assumption 3.1 and discretization (4.12) satisfies all the conditions required for Lemmas 5.1, 5.2, 5.3, then scheme (4.12) converges to the viscosity solution of equation (3.2).

Proof. This follows directly from the results in $[10,5]$.

It is also useful to note that $\left[I-(1-\theta) A^{n}\left(Q^{n}\right)\right]$ is an M-matrix [47].

Remark 5.2 (Properties of M-Matrices) An M-matrix $B$ has the properties that $B^{-1} \geq 0$ and $\operatorname{diag}\left(B^{-1}\right)>0$.

Lemma 5.4 (M-matrix) If the positive coefficient condition (4.1) is satisfied, and boundary conditions (3.10,3.13) are imposed at $S=0, S_{\max }$, then $\left[I-(1-\theta) \Delta \tau A^{n}\right]$ is an M-matrix.

Proof. Condition (4.1) implies that $\alpha_{i}^{n}, \beta_{i}^{n}, c_{i}^{n}$ in equation (4.8) are non-negative. Hence $\left[I-(1-\theta) \Delta \tau A^{n}\right]$ has positive diagonals, non-positive offdiagonals, and is diagonally dominant, so it is an M-matrix [47]. 


\subsection{Discrete Comparison Property}

It is interesting to verify that the discrete equations satisfy a discrete version of the Comparison Property (see Remark 3.1). Consider any two contingent claims $W(S, \tau), V(S, \tau)$. If $V(S, 0) \geq$ $W(S, 0)$, then by no arbitrage $V(S, \tau) \geq W(S, \tau)$. It is clearly desirable that discrete solutions of the pricing PDEs also have these discrete arbitrage inequalities.

\section{Theorem 5.2 (Discrete Arbitrage Inequality) Suppose}

(a) the discretization (4.8) satisfies the positive coefficient condition (4.2),

(b) boundary conditions are imposed at $S=0$ and $S=S_{\text {max }}$, as in equation (3.10) and (3.13), with boundary condition vector $F^{n}=\left[0, \ldots, F_{p}^{n}\right]^{\prime}$,

(c) fully implicit timestepping is used.

If $W^{n}$ and $V^{n}$ are two discrete solutions to equation (4.12), with $V^{n} \geq W^{n}$, with boundary condition vectors $F_{V}^{n+1} \geq F_{W}^{n+1}$, then $V^{n+1} \geq W^{n+1}$.

Proof. In the case of fully implicit timestepping, equation (4.12) becomes

$$
\begin{aligned}
V^{n+1} & =V^{n}+\Delta \tau \sup _{Q^{*} \in \hat{Q}}\left\{A^{n+1}\left(Q^{*}\right) V^{n+1}+D^{n+1}\left(Q^{*}\right)\right\}+\left(F_{V}^{n+1}-F_{V}^{n}\right) \\
W^{n+1} & =W^{n}+\Delta \tau \sup _{Q^{\prime} \in \hat{Q}}\left\{A^{n+1}\left(Q^{\prime}\right) W^{n+1}+D^{n+1}\left(Q^{\prime}\right)\right\}+\left(F_{W}^{n+1}-F_{W}^{n}\right)
\end{aligned}
$$

Subtracting equation (5.18) from equation (5.17), and using equation (B.2), gives

$$
\begin{aligned}
\left(V^{n+1}-W^{n+1}\right)= & \left(V^{n}-W^{n}\right)+\Delta \tau \sup _{Q^{*} \in \hat{Q}}\left\{A^{n+1}\left(Q^{*}\right) V^{n+1}+D^{n+1}\left(Q^{*}\right)\right\} \\
& -\Delta \tau \sup _{Q^{\prime} \in \hat{Q}}\left\{A^{n+1}\left(Q^{\prime}\right) W^{n+1}+D^{n+1}\left(Q^{\prime}\right)\right\}+\left(F_{V}^{n+1}-F_{V}^{n}\right)-\left(F_{W}^{n+1}-F_{W}^{n}\right) \\
\geq & \left(V^{n}-W^{n}\right)+\left(F_{V}^{n+1}-F_{V}^{n}\right)-\left(F_{W}^{n+1}-F_{W}^{n}\right) \\
& +\Delta \tau \inf _{Q \in \hat{Q}}\left\{A^{n+1}(Q)\left(V^{n+1}-W^{n+1}\right)\right\}
\end{aligned}
$$

Let $\bar{Q} \in \arg \inf _{Q \in \hat{Q}}\left\{A^{n+1}(Q)\left(V^{n+1}-W^{n+1}\right)\right\}$, so that equation (5.19) becomes

$$
\left[I-\Delta \tau A^{n+1}(\bar{Q})\right]\left(V^{n+1}-W^{n+1}\right) \geq\left(V^{n}-W^{n}\right)+\left(F_{V}^{n+1}-F_{V}^{n}\right)-\left(F_{W}^{n+1}-F_{W}^{n}\right) .
$$

By assumption $\left(V^{n}-W^{n}\right)+\left(F_{V}^{n+1}-F_{V}^{n}\right)-\left(F_{W}^{n+1}-F_{W}^{n}\right) \geq 0$ (recall that $F_{V}, F_{W}$ are identically zero except at $i=p$ where $\left.\left(F_{V}\right)_{p}^{n}=\left(V^{n}\right)_{p},\left(F_{W}\right)_{p}^{n}=\left(W^{n}\right)_{p}\right)$. Since $\left[I-\Delta \tau A^{n+1}(\bar{Q})\right]$ is an $M$ matrix (from Lemma 5.4), we have that

$$
\left(V^{n+1}-W^{n+1}\right) \geq 0 .
$$




\section{Solution of Algebraic Discrete Equations}

Although we have established that discretization (4.12) is consistent, stable and monotone, it is not obvious that this is a practical scheme, since the implicit timestepping method requires solution of highly nonlinear algebraic equations at each timestep. In this section we give two methods for solving these algebraic equations - one a relaxation scheme and the second a Newton-like (Policy) iteration.

\subsection{A Relaxation Scheme}

Writing out equation (4.12) in component form gives (for each $i<p$ )

$$
\begin{aligned}
V_{i}^{n+1}=(1-\theta) \Delta \tau \sup _{Q \in \hat{Q}}\{ & \alpha_{i}^{n+1}(Q) V_{i-1}^{n+1}+\beta_{i}^{n+1}(Q) V_{i+1}^{n+1} \\
& \left.-\left(\alpha_{i}^{n+1}(Q)+\beta_{i}^{n+1}(Q)+c_{i}^{n+1}(Q)\right) V_{i}^{n+1}+d_{i}^{n+1}(Q)\right\}+g_{i}^{n}
\end{aligned}
$$

where $g_{i}^{n}=V_{i}^{n}+\theta \Delta \tau\left[A^{n} V^{n}+D^{n}\right]_{i}$. Rearranging equation (6.1) and noting that $\alpha_{i}^{n+1}, \beta_{i}^{n+1}, c_{i}^{n+1}$ are all nonnegative, we obtain

$$
\begin{array}{r}
V_{i}^{n+1}=\sup _{Q \in \hat{Q}}\left\{(1-\theta) \Delta \tau \frac{\alpha_{i}^{n+1}(Q) V_{i-1}^{n+1}+\beta_{i}^{n+1}(Q) V_{i+1}^{n+1}+d_{i}^{n+1}(Q)}{(1+(1-\theta) \Delta \tau)\left(\alpha_{i}^{n+1}(Q)+\beta_{i}^{n+1}(Q)+c_{i}^{n+1}(Q)\right)}\right. \\
\left.+\frac{g_{i}^{n}}{(1+(1-\theta) \Delta \tau)\left(\alpha_{i}^{n+1}(Q)+\beta_{i}^{n+1}(Q)+c_{i}^{n+1}(Q)\right)}\right\} .
\end{array}
$$

Let $\hat{V}^{k+1}$ be the $(k+1)$ estimate for $V^{n+1}$. Equation $(6.2)$ can then be used as a basis for the relaxation scheme

$$
\begin{aligned}
& \hat{V}_{i}^{k+1}=\sup _{Q \in \hat{Q}}\{(1-\theta) \Delta \tau \frac{\alpha_{i}^{n+1}(Q) \hat{V}_{i-1}^{k}+\beta_{i}^{n+1}(Q) \hat{V}_{i+1}^{k}+d_{i}^{n+1}(Q)}{(1+(1-\theta) \Delta \tau)\left(\alpha_{i}^{n+1}(Q)+\beta_{i}^{n+1}(Q)+c_{i}^{n+1}(Q)\right)} \\
&\left.+\frac{g_{i}^{n}}{(1+(1-\theta) \Delta \tau)\left(\bar{\alpha}_{i}^{k}+\bar{\beta}_{i}^{k}+\bar{c}_{i}^{k}\right)}\right\} .
\end{aligned}
$$

This leads us to a constructive proof for the existence of a unique solution for the discretized equations.

Theorem 6.1 (Convergence of Relaxation) Suppose that

(a) the discretization (4.8) satisfies the positive coefficient condition (4.2),

(b) boundary conditions are imposed at $S=0$ and $S=S_{\text {max }}$, as in equation (3.10) and (3.13). 
Then a unique solution of the nonlinear equations (6.1) exists. Furthermore, the iteration scheme (6.3) is globally convergent for any initial estimate.

Proof. Writing equation (6.3) for iteration $k$, and using equation (B.4) gives

$$
\begin{aligned}
\left\|\hat{V}^{k+1}-\hat{V}^{k}\right\|_{\infty} & \leq \gamma\left\|\hat{V}^{k}-\hat{V}^{k-1}\right\|_{\infty} \\
\gamma & =\max _{i} \sup _{Q \in \hat{Q}}\left\{\frac{(1-\theta \Delta \tau)\left[\alpha_{i}^{n+1}(Q)+\beta_{i}^{n+1}(Q)\right]}{1+(1-\theta \Delta \tau)\left[\alpha_{i}^{n+1}(Q)+\beta_{i}^{n+1}(Q)+c_{i}^{n+1}(Q)\right]}\right\} .
\end{aligned}
$$

Since $\alpha_{i}^{n}(Q), \beta_{i}^{n}(Q), c_{i}^{n}(Q)$ are nonnegative for all $Q \in \hat{Q}$, we have that $\gamma<1$. Thus the scheme (6.3) is a contraction and converges to the unique solution of the discretized algebraic equations.

Remark 6.1 (Existence of solution: HJBI case) The above argument can be repeated if we replace the sup in equation (6.3) by an inf or a supinf. Hence, in all cases (HJB, or HJBI), the scheme (6.3) is a contraction. Although the solution $V^{n+1}$ is unique, the control may not be unique.

Unfortunately, this relaxation scheme is not very useful in practice. To see this consider the trivial case where $Q$ is constant. In this situation, scheme (6.3) is simply a relaxation method for the solution of a discretized parabolic PDE. Recalling the definition of the discretization parameter $h_{\min }$ in equation (4.13), this implies that the error reduction in each iteration of scheme (6.3) is

$$
\gamma \simeq \frac{1}{1+O\left(h_{\min }\right)}
$$

which is very poor as $h_{\min } \rightarrow 0$.

Remark 6.2 (Markov Chains) Consider equation (6.2) and, for simplicity, let $\theta=0$. Then write

$$
V_{i}^{n+1}=\sup _{Q_{i}^{n+1} \in \hat{Q}}\left\{P_{i, i-1}^{n+1} V_{i-1}^{n+1}+P_{i, i+1}^{n+1} V_{i+1}^{n+1}+U_{i}^{n+1}\right\}
$$

where

$$
P_{i, i-1}^{n+1}=\frac{\Delta \tau \alpha_{i}^{n+1}}{\omega_{i}}, \quad P_{i, i+1}^{n+1}=\frac{\Delta \tau \beta_{i}^{n+1}}{\omega_{i}} \quad \text { and } \quad U_{i}^{n+1}=\frac{g_{i}^{n}+\Delta \tau d_{i}^{n+1}}{\omega_{i}}
$$

with $\omega_{i}=(1+\Delta \tau)\left(\alpha_{i}^{n+1}+\beta_{i}^{n+1}+c_{i}^{n+1}\right)$. Since $0 \leq P_{i, j} \leq 1$ and $\sum_{j} P_{i, j}<1$, we can identify the $P_{i, j}$ as discounted risk neutral transition probabilities. Hence, at each timestep, we can consider equation (6.6) as the solution of an infinite horizon controlled Markov chain [27]. We will refer to equation (6.6) as the Markov chain form of the discretized equations. Note that the Markov chain form necessarily puts some terms involving the control parameter in the denominator of the probabilities $P_{i, j}$. This can cause some complications, and various methods have been suggested to remedy this problem [27]. However, this rearrangement is somewhat unnatural from the PDE point of view. 
Remark 6.3 (Value Iteration) We can view the iteration (6.3) as similar to the familiar value iteration in stochastic control [27]. In this context, the problem is usually formulated as a discrete Markov chain, as in Remark 6.2.

\subsection{Policy Iteration}

It would seem desirable to have a scheme which converged in one iteration if $Q$ is constant. This leads us to the following iterative scheme.

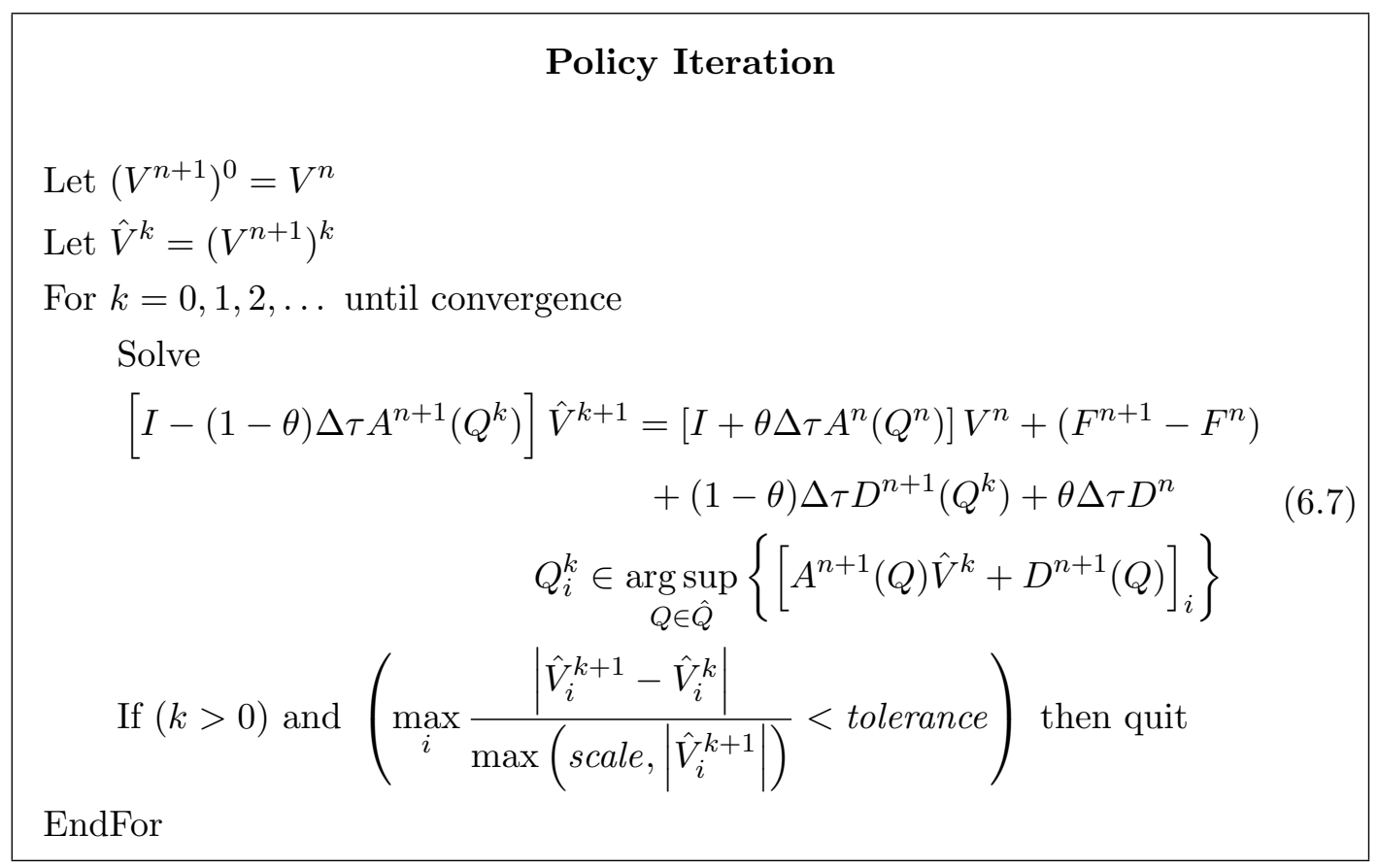

The term scale in scheme (6.7) is used to ensure that unrealistic levels of accuracy are not required when the value is very small. Typically, scale $=1$ for options priced in dollars.

Some manipulation of algorithm (6.7) results in

$$
\begin{array}{r}
{\left[I-(1-\theta) \Delta \tau A^{n+1}\left(Q^{k}\right)\right]\left(\hat{V}^{k+1}-\hat{V}^{k}\right)=(1-\theta) \Delta \tau\left[\left(A^{n+1}\left(Q^{k}\right) \hat{V}^{k}+D^{n+1}\left(Q^{k}\right)\right)\right.} \\
\left.-\left(A^{n+1}\left(Q^{k-1}\right) \hat{V}^{k}+D^{n+1}\left(Q^{k-1}\right)\right)\right] .
\end{array}
$$

We can also write equation (6.8) as

$$
\left[I-(1-\theta) \Delta \tau A^{n+1}\left(Q^{k}\right)\right]\left(\hat{V}^{k+1}-\hat{V}^{k}\right)=-R^{k},
$$

where the residual $R$ vector is

$$
R^{k}=\hat{V}^{k}-V^{n}-\left[(1-\theta) \Delta \tau\left(A^{n+1}\left(Q^{k}\right) \hat{V}^{k}+D^{n+1}\left(Q^{k}\right)\right)+H^{n}\right]
$$


with

$$
H_{n}=\theta \Delta \tau\left(A^{n}\left(Q^{n}\right) V^{n}+D^{n}\right)+\left(F^{n+1}-F^{n}\right) .
$$

In order to prove the convergence of Algorithm (6.7), we first need an intermediate result.

Lemma 6.1 (Sign of RHS of Equation (6.8)) If $A^{n+1}\left(Q^{k}\right) \hat{V}^{k}$ is given by equation (4.8), with the control parameter determined by

$$
Q_{i}^{k} \in \underset{Q \in \hat{Q}}{\arg \sup }\left\{\left[A^{n+1}(Q) \hat{V}^{k}+D^{n+1}(Q)\right]_{i}\right\},
$$

then every element of the right hand side of equation (6.8) is nonnegative, that is,

$$
\left[\left(A^{n+1}\left(Q^{k}\right) \hat{V}^{k}+D^{n+1}\left(Q^{k}\right)\right)-\left(A^{n+1}\left(Q^{k-1}\right) \hat{V}^{k}+D^{n+1}\left(Q^{k-1}\right)\right)\right]_{i} \geq 0
$$

Proof. Recall that $Q^{k}$ is selected so that

$$
A^{n+1}\left(Q^{k}\right) \hat{V}^{k}+D^{n+1}\left(Q^{k}\right)=\sup _{Q \in \hat{Q}}\left\{A^{n+1}(Q) \hat{V}^{k}+D^{n+1}(Q)\right\} .
$$

for given $\hat{V}^{k}$. Hence, any other choice of coefficients, for example

$$
A^{n+1}\left(Q^{k-1}\right) \hat{V}^{k}+D^{n+1}\left(Q^{k-1}\right)
$$

cannot exceed equation (6.14).

It is now easy to show that iteration (6.7) always converges.

Theorem 6.2 (Convergence of Iteration (6.7)) Provided that the conditions required for Lemmas 6.1 and 5.4 are satisfied, then the nonlinear iteration (6.7) converges to the unique solution of equation (4.12) for any initial iterate $\hat{V}^{0}$. Moreover, the iterates converge monotonically.

Proof. Given Lemmas 6.1 and 5.4, the proof of this result is similar to the proof of convergence given in [38]. We give a brief outline of the steps in this proof, and refer readers to [38] for details. A straightforward maximum analysis of scheme (6.7) can be used to bound $\left\|\hat{V}^{k}\right\|_{\infty}$ independent of iteration $k$. From Lemma 6.1, we have that the right hand side of equation (6.8) is nonnegative. Noting that $\left[I-(1-\theta) \Delta \tau A^{n+1}\left(Q^{k}\right)\right]$ is an M-matrix (from Lemma 5.4) and hence $\left[I-(1-\theta) \Delta \tau A^{n+1}\left(Q^{k}\right)\right]^{-1} \geq 0$, it is easily seen that the iterates form a bounded non-decreasing sequence. In addition, if $\hat{V}^{k+1}=\hat{V}^{k}$ the residual is zero. Hence the iteration converges to a solution. It follows from the M-matrix property of $\left[I-(1-\theta) \Delta \tau A^{n+1}\left(Q^{k}\right)\right]$ that the solution is unique.

The above proof can be repeated with the sup replaced by inf in equation (6.7). 
Remark 6.4 (Q Dependent Discretizations) Note that we obtain convergence for the case of $Q$ - dependent discretizations, even if the discrete equations, regarded as a function of the control $Q$, are discontinuous. This is discussed in [49].

Remark 6.5 (Policy Iteration) Iteration (6.7) is essentially the well known policy iteration in stochastic control [42]. It differs slightly in that we do not use the Markov chain rearrangement of the discrete equations, as in equation (6.6). Hence, the iteration sequence will be different than the classical policy iteration (a different local control problem is solved at each node), but the convergence result is the same. Since we do not rearrange the discrete equations into the Markov chain form, we do not have the difficulties associated with control parameters appearing in the denominator of the discrete equations, as discussed in Remark 6.2.

Remark 6.6 (Equivalence of Iteration (6.7) and Newton Iteration) Suppose that that there is a single control at each node $Q_{i}$, and that the sup control is unconstrained. Then, from equation (6.10), assuming that the discrete equations are differentiable, we have

$$
\frac{\partial R_{i}^{k}}{\partial \hat{V}_{j}^{k}}=\frac{\partial R_{i}^{k}}{\partial Q_{i}^{k}} \frac{\partial Q_{i}}{\partial \hat{V}_{j}^{k}}+\left(\delta_{i j}-(1-\theta) \Delta \tau A_{i j}^{n+1}\left(Q^{k}\right)\right) .
$$

But

$$
\frac{\partial R_{i}^{k}}{\partial Q_{i}^{k}}=0
$$

since $Q_{i}^{k}$ is locally optimal. Hence the iteration

$$
\left[I-(1-\theta) \Delta \tau A^{k}\right]\left(\hat{V}^{k+1}-\hat{V}^{k}\right)=-R^{k},
$$

which is equivalent to iteration (6.7), is a Newton iteration. Of course, in general the coefficients may not be differentiable, and the control parameters are constrained. Nevertheless, as discussed in [40, 39, 42], we may view iteration (6.7) as a Newton-like iteration (quadratic convergence when close to solution).

Remark 6.7 (Policy Iteration: HJBI Equation) For the case of the HJBI equation (problems with a sup inf, equation (3.8)), it is not clear when iteration (6.7) can be expected to converge. The convergence argument breaks down in this case, since we cannot expect Lemma 6.1 to hold. However, as discussed in [36], we can also interpret policy iteration as a form of Newton-like iteration, for the case of a finite set of controls. In this case, we can expect convergence, even for the stochastic game case, if the initial estimate is sufficiently close to the solution.

\section{Piecewise Constant Policies}

The relaxation scheme (also known as value iteration) (6.3) from the previous section is globally convergent to the unique solution of the discretized equations for both HJB and HJBI equations. However, the convergence rate becomes unacceptably slow as the grid size is reduced. 
The policy iteration scheme (6.7) is globally convergent but only for HJB equations. Since this method can be regarded as a Newton-like iteration, convergence will typically be very rapid if the initial estimate is sufficiently close to the solution. In typical option pricing problems, where we have the solution from the previous timestep as the initial guess, convergence generally occurs in $2-3$ iterations if six digit accuracy is specified.

Unfortunately, there are examples where the convergence rates can be slow. In [42], an example with discrete controls is constructed whereby the iteration (beginning from the zero state), takes $R-1$ steps, where $R$ is the number of states (which would correspond to nodes in our case). In some cases, it may also be a nontrivial problem to solve the local control problem (6.12). This may be especially difficult if jump processes are modelled, which results in a controlled partial integrodifferential equation (PIDE) [24]. In addition, the policy iteration scheme does not guarantee global convergence of (6.7) for HJBI equations. Indeed there are pathological cases where policy iteration does not converge for these problems (c.f. [48]). This has led to the development of several variants of Newton iteration which attempt to ensure global convergence for these problems $[45,14,46]$.

In this section we consider an alternate timestepping method, one which is guaranteed to converge to the viscosity solution, does not have timestep sizes linked to the mesh size (which precludes explicit methods), and does not require solution of nonlinear equations at each step.

\subsection{An Informal Approach}

The basic idea behind the piecewise constant policy approximation is intuitively appealing. Suppose an agent is allowed to make changes in the control only at discrete forward times $t_{i}, i=1, \ldots, L$. We will also assume that the agent can choose from only a finite number of controls, that is, all possible control choices can be enumerated $Q^{m}, m=1, \ldots, m_{\max }$ (for example $m_{\max }=2$ in Example 2.1 and $m_{\max }=8$ in Example 2.2 - double if we are looking at American options). In the case that the control variables are continuous, we approximate the control by a finite set of piecewise constant policies.

Let $\tau^{i}=T-t_{i}$ and $\mathcal{V}_{m}$ be the solution to

$$
\left(\mathcal{V}_{m}\right)_{\tau}=\mathcal{L}^{Q^{m}} \mathcal{V}_{m}+d\left(Q^{m}\right)
$$

where $\mathcal{L}^{Q^{m}}$ denotes the operator (3.1) for a fixed value of $Q^{m}$. In other words, $\mathcal{V}_{m}$ is the solution to the optimal control problem with the trivial constant policy $Q^{m}$. At $t=T, \tau=0$, we set

$$
\mathcal{V}_{m}(S, 0)=\text { Option Payoff } ; \forall m \text {. }
$$

Now suppose the agent is at $t=t_{L}$, the last decision time before the contract expiry at $t=T$. In order to determine the optimal policy at $\tau=T-t_{L}=\tau^{L}$, the agent examines all possible choices of the the policy, and chooses the policy which maximizes the value of the contract. This is simply done by solving

$$
\left(\mathcal{V}_{m}\right)_{\tau}=\mathcal{L}^{Q^{m}} \mathcal{V}_{m}+d^{m} \text { with } d^{m}=d\left(Q^{m}, S, \tau\right)
$$

from $\tau=0$ to $\tau=\tau^{L}$ for all $m=1, \ldots, m_{\max }$. The optimal value is then determined simply from

$$
V^{o p t}\left(S, \tau^{L}\right)=\max _{m} \mathcal{V}_{m}\left(S, \tau^{L}\right)
$$


We then set

$$
\mathcal{V}_{m}\left(S, \tau^{L}+\epsilon\right)=V^{o p t}\left(S, \tau^{L}\right) ; \epsilon>0, \epsilon \ll 1 ; \forall m
$$

and repeat the above procedure at $\tau=\tau^{L-1}$, and so on. If the times between decision dates $t_{i}$ are small, and we have used a large enough sample of the policy space $Q$, then this should be a good approximation to the original control problem (3.2).

\subsection{A Formal Approach}

More precisely, consider the following algorithm. For simplicity, we consider only fully implicit timestepping.

Piecewise Constant Policy Timestepping
$\begin{aligned} & V^{0}=\text { Option Payoff } \\ & \text { For } n=0, \ldots, \quad / / \text { Timestep Loop } \\ & \mathcal{V}_{i, m}^{n}=V_{i}^{n} ; i=1, \ldots, p \quad m=1, \ldots, m_{\max } \\ & \text { For } m=1, \ldots, m_{\text {max }} \\ & \quad \text { Solve } \\ & \quad\left(I-\Delta \tau A^{n+1}\left(Q^{m}\right)\right) \mathcal{V}_{m}^{n+1 / 2}=\mathcal{V}_{m}^{n}+\Delta \tau D^{n+1}\left(Q^{m}\right) \\ & \text { EndFor } \\ & V_{i}^{n+1}=\max _{j} \mathcal{V}_{i, j}^{n+1 / 2} ; i=1, \ldots, p-1 \\ & V_{p}^{n+1}=F_{p}^{n+1} \\ & \text { EndFor } \quad / / \quad \text { End Timestep Loop }\end{aligned}$

Note that we have used a slightly different time discretization here compared with equation (4.12), with the boundary condition updated explicitly.

We will now verify that that this scheme satisfies the sufficient conditions for convergence.

Lemma 7.1 (Stability of Scheme (7.6)) If the discretization (4.8) satisfies the same conditions as for Lemma 5.1, then the same stability result (Lemma 5.1) holds for piecewise constant policy timestepping.

Proof. This follows using the same maximum analysis as used in the proof of Lemma 5.1.

Showing consistency is a more challenging problem. In order to determine if the consistency condition (5.2) is satisfied, we need to eliminate $\mathcal{V}_{m}^{n+1 / 2}$ from equation (7.6). Let

$$
\begin{aligned}
\mathcal{V}_{m}^{n+1 / 2} & =H_{m}\left(V^{n}\right) \\
& =\left[I-\Delta \tau A^{n+1}\left(Q^{m}\right)\right]^{-1}\left[V^{n}+\Delta \tau D^{n+1}\left(Q^{m}\right)\right] .
\end{aligned}
$$


Eliminating $\mathcal{V}_{m}^{n+1 / 2}$ in equation (7.6) gives

$$
V_{i}^{n+1}=\max _{j}\left(\left[H_{j}\left(V^{n}\right)\right]_{i}\right)
$$

or equivalently

$$
G_{i}^{n+1}\left(h, V_{i}^{n+1},\left\{V_{j}^{n}\right\}\right)= \begin{cases}\frac{V_{i}^{n+1}-\max _{j}\left(\left[H_{j}\left(V^{n}\right)\right]_{i}\right)}{\Delta \tau}, & i<p \\ V_{p}^{n+1}-F_{p}^{n+1}, & i=p .\end{cases}
$$

It turns out to be non-trivial to show consistency. In [8], mollification techniques are used to show consistency for the case where the PDE coefficients are time independent. In [44], a complex argument is used to show consistency for first order problems.

In fact, we can avoid this difficulty by noting that in [25], convergence of the piecewise constant policy method is proven using probabilistic methods, in the context of the dynamic programming principle. The probabilistic solution is the viscosity solution of the related HJB equation. In addition, in [25], it is shown that the constant control diffusion operator is consistent (over small timesteps) with the dynamic programming operator for fixed control (Lemma 5.10 in [25]). Hence, if our discrete diffusion operator is consistent with equation (7.1), then it is also consistent with the dynamic programming operator (for fixed controls). Thus we can regard a discretization of equation (7.1) as a discretization of the dynamic programming operator, and we know from [25] that the piecewise constant policy algorithm (using the dynamic programming operator) converges to the viscosity solution.

This leads us to the following definition of consistency for piecewise constant policy methods.

Definition 7.1 (Consistency Requirement for Piecewise Constant Policy Schemes [25, 26]) Given a smooth function $\phi$, then consistency is defined as

$$
\lim _{h \rightarrow 0}\left|\frac{\phi_{i}^{n+1}-\phi_{i}^{n}}{\Delta \tau}-\left[A^{n+1}\left(Q^{m}\right) \phi^{n+1}+\Delta \tau D^{n+1}\left(Q^{m}\right)\right]_{i}-\left[\phi_{\tau}-\mathcal{L}^{Q^{m}} \phi-d\left(Q^{m}\right)\right]_{i}^{n+1}\right|=0
$$

for all fixed $Q^{m}$ where $(\Delta S)_{\max }=C_{1} h, \Delta \tau=C_{2} h$ and $C_{1}, C_{2}$ are independent of $h$.

Lemma 7.2 (Consistency for Piecewise Constant Policy Schemes (7.6)) Discretization (7.6), where $\mathcal{L}^{Q^{m}}$ is given by equation (4.1), satisfies the consistency requirement given in Definition 7.1.

Proof. This follows from equation (4.5).

Lemma 7.3 (Monotonicity of Scheme (7.6)) If the discretization (4.8) satisfies the positive coefficient condition (4.2), with boundary conditions at $S=0$ and $S=S_{\max }$, as in equation (3.10) and (3.13), then discretization (7.6) is monotone as defined in Definition 5.3.

Proof. Recall that

$$
G_{i}^{n+1}\left(h, V_{i}^{n+1},\left\{V_{j}^{n}\right\}\right)=\frac{V_{i}^{n+1}-\max _{m}\left(\left[H_{m}\left(V^{n}\right)\right)\right]_{i}}{\Delta \tau} .
$$


and

$$
\left.\left[H_{m}\left(V^{n}\right)\right)\right]_{i}=\left[\left[I-\Delta \tau A^{n+1}\left(Q^{m}\right)\right]^{-1}\left[V^{n}+\Delta \tau D^{n+1}\left(Q^{m}\right)\right]\right]_{i} .
$$

Since $\left[I-\Delta \tau A^{n+1}\left(Q^{m}\right)\right]$ is an $M$ matrix (from Lemma 5.4), we have that $\left[I-\Delta \tau A^{n+1}\left(Q^{m}\right)\right]^{-1} \geq 0$, and so

$$
G_{i}^{n+1}\left(h, V_{i}^{n+1},\left\{V_{j}^{n}+\epsilon_{j}^{n}\right\}\right)-G_{i}^{n+1}\left(h, V_{i}^{n+1},\left\{V_{j}^{n}\right\}\right) \leq 0
$$

Finally, we state our convergence result for scheme (7.6).

Theorem 7.1 (Convergence to the Viscosity Solution of Method (7.6)) Provided that the original HJB satisfies Assumption 3.1 and discretization (4.12) satisfies all the conditions required for Lemmas 7.1, 7.2, 7.3, then scheme (7.6) converges to the viscosity solution of equation (3.2).

Proof. This follows directly from the results in [25].

\section{Numerical Examples}

In this section, we will use the discretization methods discussed in Section 4. We will use both the implicit control method (Section 4) and the piecewise constant control timestepping method (Section 7). These algorithms will be demonstrated on two problems: unequal borrowing and lending rates, and stock borrowing fees.

\subsection{Unequal Borrowing Lending Rates}

\subsubsection{Implicit Control}

Table 1 shows the data used for the unequal borrowing/lending example described in Section 2.1. The payoff is assumed to be a European straddle

$$
\text { Straddle Payoff }=\max (S-K, 0)+\max (K-S, 0) .
$$

Table 2 shows the results of a convergence study for the this problem. We include a test of Crank-Nicolson timestepping, even though the timesteps violate the monotonicity condition (5.5). In this example, we can only prove that the fully implicit method converges to the viscosity solution. However, in this case, the Crank-Nicolson examples also converge to the viscosity solution. The Crank Nicolson timestepping incorporates the modification suggested in [41], to improve convergence for non-smooth payoffs. As noted previously, use of a method which violates the monotonicity conditions cannot be recommended in general [38].

Note that the total number of nonlinear iterations is always twice the number of timesteps, which is the minimum possible in Algorithm 6.7. This indicates that the nonlinearity is not too 


\begin{tabular}{ll}
\hline Parameter & Value \\
\hline \hline$\sigma$ & .30 \\
$T$ & 1.0 years \\
$r_{b}$ & .05 \\
$r_{l}$ & .03 \\
$K$ & 100 \\
Convergence Tolerance tol (Algorithm 6.7$)$ & $10^{-6}$ \\
\hline
\end{tabular}

TABLE 1: Unequal borrowing/lending rate example (Section 2.1).

\begin{tabular}{|c|c|c|c|c|c|}
\hline Nodes & Timesteps & Nonlinear iterations & Option value & Change & Ratio \\
\hline \multicolumn{5}{|c|}{ Fully Implicit: Short } \\
\hline 101 & 100 & 200 & 24.02047 & & \\
401 & 200 & 400 & 24.05001 & .02954 & \\
801 & 400 & 800 & 24.06137 & .01136 & 2.6 \\
\hline \multicolumn{5}{|c|}{ Crank-Nicolson: Short } \\
\hline 101 & 100 & 200 & 24.0512 & \\
201 & 200 & 400 & 24.06554 & .01432 & \\
401 & 400 & 800 & 24.06917 & .00363 & 3.9 \\
801 & 800 & 1600 & 24.07008 & .00091 & 4.0 \\
\hline \multicolumn{5}{|c|}{ Fully Implicit: Long } & \\
\hline 101 & 100 & 200 & 23.05854 & & \\
201 & 200 & 400 & 23.08880 & .03026 & \\
401 & 400 & 800 & 23.10029 & .01141 & 2.7 \\
801 & 800 & 1600 & 23.10511 & .00481 & 2.4 \\
\hline \multicolumn{5}{|c|}{ Crank-Nicolson: Long } \\
\hline 101 & 100 & 200 & 23.08893 & & \\
201 & 200 & 400 & 23.10414 & .01525 & \\
401 & 400 & 800 & 23.10800 & .00386 & 4.0 \\
801 & 800 & 1600 & 23.10897 & .00097 & 4.0 \\
\hline
\end{tabular}

TABLE 2: Convergence for fully implicit and Crank-Nicolson timestepping using the implicit control method (Section 4) and the discrete equations solved using the policy iteration method (6.7). Unequal borrowing/lending rate example as in Section 2.1. Crank Nicolson incorporates the modification suggested in [41]. Input parameters are given in Table 1. Straddle payoff (8.1), option values reported at $S=100, t=0$. 


\begin{tabular}{|c|c|c|c|c|}
\hline Nodes & Timesteps & Option value & Change & Ratio \\
\hline \multicolumn{5}{|c|}{ Fully Implicit: Short } \\
\hline 101 & 100 & 24.01163 & & \\
201 & 200 & 24.04550 & .03387 & \\
401 & 400 & 24.05908 & .01358 & 2.5 \\
801 & 800 & 24.06502 & .00594 & 2.3 \\
\hline \multicolumn{5}{|c|}{ Crank-Nicolson: Short } \\
\hline 101 & 100 & 24.04652 & & \\
201 & 200 & 24.06318 & .01666 & \\
401 & 400 & 24.06799 & .00481 & 3.5 \\
801 & 800 & 24.06949 & .00150 & 3.2 \\
\hline \multicolumn{5}{|c|}{ Fully Implicit: Long } \\
\hline 101 & 100 & 23.06752 & \\
201 & 200 & 23.09338 & .02586 & \\
401 & 400 & 23.10261 & .00923 & 2.8 \\
801 & 800 & 23.10628 & .00366 & 2.5 \\
\hline \multicolumn{5}{|c|}{ Crank-Nicolson: Long } \\
\hline 101 & 100 & 23.09371 & \\
201 & 200 & 23.10653 & .01282 & \\
401 & 400 & 23.10919 & .00266 & 4.8 \\
801 & 800 & 23.10957 & .00038 & 7.0 \\
\hline
\end{tabular}

TABLE 3: Convergence for fully implicit and Crank-Nicolson timestepping using the piecewise constant policy method (Section 7) with timestepping scheme (7.6). Unequal borrowing/lending rate example as in Section 2.1. Crank Nicolson incorporates the modification suggested in [41]. Input parameters are given in Table 1. Straddle payoff (8.1), option values reported at $S=100, t=0$.

severe. This seems to be typical of HJB problems in finance, which often have bang-bang type optimal controls. The same behavior was noted in $[3,22,38]$. In contrast to the numerical results in [38] for uncertain volatility models, we seem to obtain smooth second order convergence for the Crank-Nicolson case.

\subsubsection{Piecewise Constant Policy}

Table 3 gives the results for a convergence study of the same problem, using the piecewise constant policy timestepping of Section 7. At each timestep, we solve two problems with constant controls $\left\{r_{l}, r_{b}\right\}$, and use Algorithm (7.6) to advance the solution.

Again, both fully implicit and Crank-Nicolson methods converge to the viscosity solution, although convergence can only be proved for the fully implicit method. In this case, however, the convergence of Crank-Nicolson is somewhat erratic. However, this is not unexpected, since the application of the max operation at the end of each timestep in Algorithm 7.6 can be expected to generate non-smoothness, which is known to be a problem with Crank-Nicolson.

Figure 1 shows the option value for the short and long case, for the unequal borrowing/lending problem. Figure 2 includes the results for the case where $r_{l}=r_{b}=.03$ and $r_{l}=r_{b}=.05$. Note 


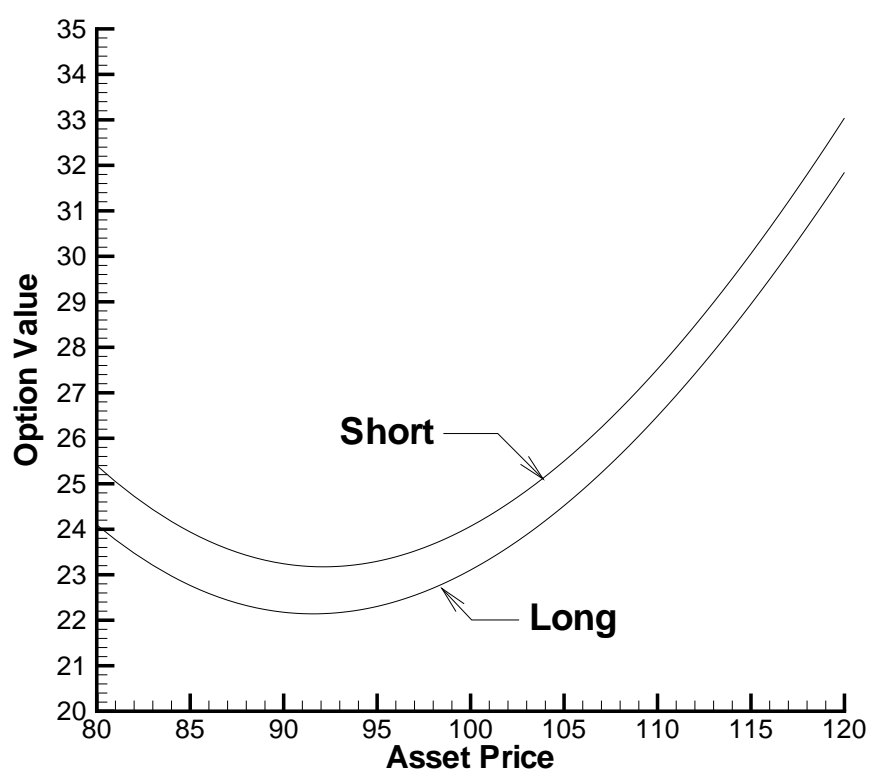

Figure 1: The option value for a straddle, data in Table 1.

that the long/short solutions to equation (2.7) are outside the envelop of solutions with constant $r_{l}=r_{b}$ set to the maximum and minimum extreme values. Figure 2 clearly illustrates the nonlinear nature of equation (2.7).

\subsection{Stock Borrowing Fees}

Table 4 shows the data used for the stock borrowing fee problem described in Section 2.2. Note that all the data is the same as in Table 1, with the exception that we have specified $r_{f}$. A straddle payoff (8.1) was also specified.

\subsubsection{European Case}

Tables 5 and 6 show the results for a convergence study of this problem, using both the implicit control method (Section 4) and piecewise constant policy timestepping (Section 7). In the case of the piecewise constant policy, since $V \geq 0$ in this case (see equation (2.11)), $q_{2}=r_{l}$ if short, and $q_{2}=r_{b}$ if long, so that we only need to solve for the four possible combinations of constant controls $q_{1}=\left\{r_{l}, r_{b}\right\}, q_{1}=\{0,1\}$ at each timestep.

In all cases, Crank-Nicolson timestepping converges to the viscosity solution. As well, for the implicit control case, the number of iterations required for Algorithm 6.7 is the minimum possible. Again, Crank-Nicolson convergence is erratic for the piecewise constant policy method. 


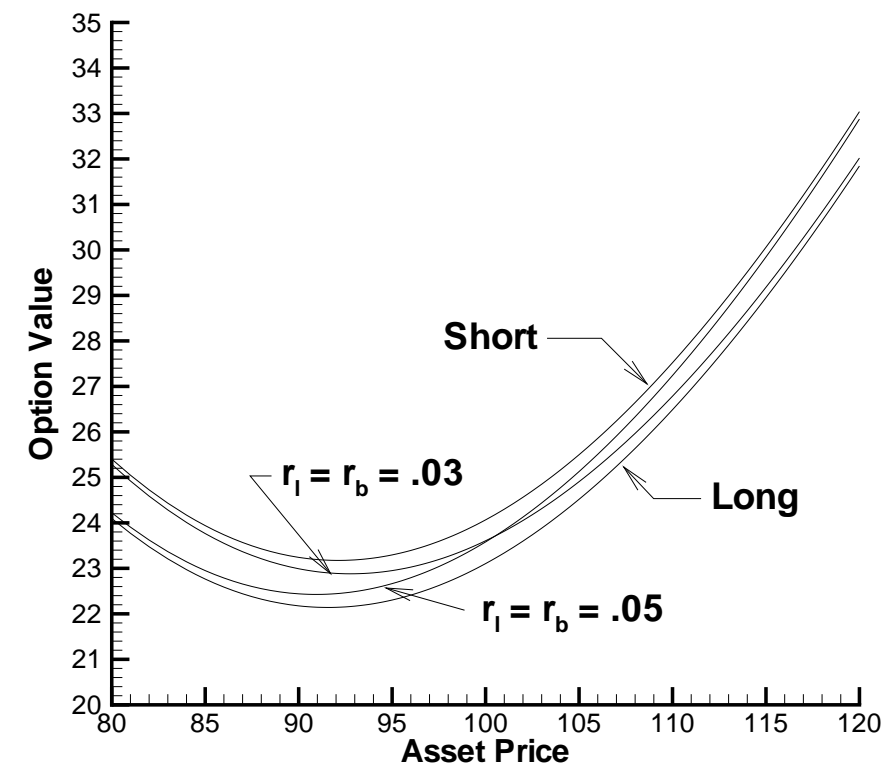

Figure 2: The option value for a straddle, data in Table 1. Also shown are the option values for $r_{l}=r_{b}=.05$ and $r_{l}=r_{b}=.03$. Note that for constant interest rates, the value of the straddle at $S=K=100$ is very insensitive to changes in interest rate.

\begin{tabular}{ll}
\hline Parameter & Value \\
\hline \hline$\sigma$ & .30 \\
$T$ & 1.0 years \\
$r_{b}$ & .05 \\
$r_{l}$ & .03 \\
$r_{f}$ & .004 \\
$K$ & 100 \\
Convergence Tolerance tol (Algorithm 6.7) & $10^{-6}$ \\
Penalty term $\epsilon$ (equation 2.15) & $10^{-6} \Delta \tau_{0}$ \\
$\Delta \tau_{0}$ & Initial timestep, coarse grid \\
\hline
\end{tabular}

TABLE 4: Stock Borrowing fee (Section 2.2) example. 


\begin{tabular}{|c|c|c|c|c|c|}
\hline Nodes & Timesteps & Nonlinear iterations & Option value & Change & Ratio \\
\hline \multicolumn{5}{|c|}{ Fully Implicit: Short } \\
\hline 101 & 100 & 200 & 24.08463 & & \\
401 & 200 & 400 & 24.11412 & .02950 & \\
801 & 400 & 800 & 24.12549 & .01137 & 2.6 \\
\hline \multicolumn{5}{|c|}{ Crank-Nicolson: Short } \\
\hline 101 & 100 & 200 & 24.11552 & & \\
201 & 200 & 400 & 24.12972 & .01420 & \\
401 & 400 & 800 & 24.13333 & .00361 & 3.9 \\
801 & 800 & 1600 & 24.13423 & .00090 & 4.0 \\
\hline \multicolumn{5}{|c|}{ Fully Implicit: Long } \\
\hline 101 & 100 & 200 & 22.63266 & & \\
201 & 200 & 400 & 22.66339 & .03073 & \\
401 & 400 & 800 & 22.67514 & .01175 & 2.6 \\
801 & 800 & 1600 & 22.68009 & .00495 & 2.4 \\
\hline \multicolumn{5}{|c|}{ Crank-Nicolson: Long } \\
\hline 101 & 100 & 200 & 22.66412 & & \\
201 & 200 & 400 & 22.67927 & .01515 & \\
401 & 400 & 800 & 22.68312 & .00385 & 3.9 \\
801 & 800 & 1600 & 22.68408 & .00096 & 4.0 \\
\hline
\end{tabular}

TABLE 5: Convergence for fully implicit and Crank-Nicolson timestepping using the implicit control method (Section 4) and the discrete equations solved using the policy iteration (6.7). Stock borrowing fee example as described in Section 2.2. Crank Nicolson incorporates the modification suggested in [41]. Input parameters are given in Table 4. Straddle payoff (8.1), option values reported at $S=100$, $t=0$. 


\begin{tabular}{|l|l|l|l|l|}
\hline Nodes & Timesteps & Option value & Change & Ratio \\
\hline \multicolumn{5}{|c|}{ Fully Implicit: Short } \\
\hline 101 & 100 & 24.07437 & & \\
201 & 200 & 24.10889 & .03452 & \\
401 & 400 & 24.12284 & .01395 & 2.5 \\
801 & 800 & 24.12896 & .00612 & 2.3 \\
\hline \multicolumn{5}{|c|}{ Crank-Nicolson: Short } \\
\hline 101 & 100 & 24.11006 & .01693 & \\
201 & 200 & 24.12699 & .01649 & 3.4 \\
401 & 400 & 24.13196 & .00497 \\
801 & 800 & 24.13350 & .00154 & 3.2 \\
\hline \multicolumn{5}{|c|}{ Fully Implicit: Long } \\
\hline 101 & 100 & 22.64142 & \\
201 & 200 & 22.66787 & .02645 & \\
401 & 400 & 22.67741 & .00954 & 2.8 \\
801 & 800 & 22.68123 & .00382 & 2.5 \\
\hline \multicolumn{5}{|c|}{ Crank-Nicolson: Long } \\
\hline 101 & 100 & 22.66884 & \\
201 & 200 & 22.68163 & .01279 & \\
401 & 400 & 22.68430 & .00267 & 4.8 \\
801 & 800 & 22.68467 & .00037 & 7.2 \\
\hline
\end{tabular}

TABLE 6: Convergence for fully implicit and Crank-Nicolson timestepping using the piecewise constant policy method (Section 7) with timestepping scheme (7.6). Crank Nicolson incorporates the modification suggested in [41]. Stock borrowing fee example as described in Section 2.2. Input parameters are given in Table 4. Straddle payoff (8.1), option values reported at $S=100, t=0$. 


\begin{tabular}{|c|c|c|c|c|c|}
\hline Nodes & Timesteps & Nonlinear iterations & Option value & Change & Ratio \\
\hline \multicolumn{5}{|c|}{ Fully Implicit: Long } \\
\hline 101 & 100 & 219 & 23.01909 & & \\
201 & 200 & 440 & 23.05586 & .03677 & \\
401 & 400 & 879 & 23.07092 & .01506 & 2.4 \\
801 & 800 & 1707 & 23.07761 & .00669 & 2.3 \\
\hline
\end{tabular}

TABLE 7: Convergence for fully implicit timestepping using implicit controls. (Section 4) and the discrete equations solved using the policy iteration method (6.7). Stock borrowing fee example, with American early exercise, as described in Section 2.4 (HJBI equation). Input parameters are given in Table 4. Straddle payoff (8.1), option values reported at $S=100, t=0$.

\subsubsection{American Case: HJBI Equation}

In the following sections, we will consider a long position with stock borrowing fees, and American early exercise. This corresponds to the HJBI equation (stochastic game) given in Example 2.4 from Section 2.

\subsubsection{HJBI Example: Implicit Control}

Table 7 shows a convergence study for the long borrowing fee example, with an American early exercise feature, using implicit controls. In this case, we have no proof that the iterative Algorithm 6.7 is globally convergent, although as discussed in Remark 6.7, we can expect convergence for bang-bang controls if the timestep is sufficiently small (a good estimate of the solution from the previous timestep).

\subsubsection{HJBI Example: Piecewise Constant Controls}

Table 8 shows a convergence study of the solution to the HJBI problem, but this time we evaluate the American constraint in explicit fashion at the end of each timestep. This corresponds to using implicit controls for the inf control in equation (2.15), and a piecewise constant policy for the sup control. This, of course, corresponds to the standard Bermudan approximation of American options in finance. Comparing Tables 7 and 8, we see that the explicit evaluation of the American constraint is slightly less work than the implicit control approach, and the convergence appears to be similar. Note that using the explicit American constraint results in a method where the iteration (6.7) is guaranteed to converge.

Table 9 shows the results for the same American problem, this time using piecewise constant policy for the inf controls in equation (2.15). This means that we solve four separate one-dimensional problems at each timestep, one for each possible control combination. Within each constant control problem, the American constraint is handled implicitly.

Table 10 shows a convergence study using piecewise constant policy timestepping for all the controls, i.e. four problems are solved for each possible inf control combination, and the American constraint is applied explicitly. The results are very similar to those in Table 9. Note that in this case, each timestep requires the solution of four one-dimensional linear PDEs. If we define a unit of work as the work required for a single one-dimensional PDE solve, or for one iteration of a 


\begin{tabular}{|c|c|c|c|c|c|}
\hline Nodes & Timesteps & Nonlinear iterations & Option value & Change & Ratio \\
\hline \multicolumn{5}{|c|}{ Fully Implicit: Long } \\
\hline 101 & 100 & 200 & 23.01051 & & \\
401 & 200 & 400 & 23.05119 & .04068 & \\
801 & 400 & 800 & 23.06842 & .01723 & 2.4 \\
& 800 & 1600 & 23.07632 & .00790 & 2.2 \\
\hline
\end{tabular}

TABLE 8: Convergence for fully implicit timestepping using an explicit evaluation of the American constraint. (Section 4) and the discrete equations solved using the policy iteration method (6.7). Stock borrowing fee example as described in Section 2.4 (HJBI equation). Input parameters are given in Table 4. Straddle payoff (8.1), option values reported at $S=100, t=0$. American early exercise.

\begin{tabular}{|c|c|c|c|c|c|}
\hline Nodes & Timesteps & Nonlinear iterations & Option value & Change & Ratio \\
\hline \multicolumn{5}{|c|}{ Fully Implicit: Long } \\
201 & 100 & 1233 & 23.02816 & & \\
401 & 200 & 2536 & 23.06049 & .03233 & \\
801 & 400 & 5209 & 23.07326 & .01277 & 2.5 \\
& 800 & 10737 & 23.07882 & .00556 & 2.3 \\
\hline
\end{tabular}

TABLE 9: Convergence for fully implicit timestepping using an implicit evaluation of the American constraint, using the piecewise constant policy method (Section 7) with timestepping scheme (7.6). Stock borrowing fee example, American early exercise, as described in Section 2.4 (HJBI equation). Input parameters are given in Table 4. Straddle payoff (8.1), option values reported at $S=100$, $t=0$. Note that the number of iterations is the total number for all four problems solved each timestep. American constraint handled implicitly. 


\begin{tabular}{|c|c|c|c|c|}
\hline Nodes & Timesteps & Option value & Change & Ratio \\
\hline \multicolumn{5}{|c|}{ Fully Implicit: Long } \\
\hline 101 & 100 & 23.01958 & & \\
201 & 200 & 23.05582 & .03624 & \\
401 & 400 & 23.07077 & .01495 & 2.4 \\
801 & 800 & 23.07751 & .00674 & 2.2 \\
\hline
\end{tabular}

TABLE 10: Convergence for fully implicit timestepping using an explicit evaluation of the American constraint, using the piecewise constant policy method (Section 7) with timestepping scheme (7.6). Stock borrowing fee example, American early exercise, as described in Section 2.4 (HJBI equation). Input parameters are given in Table 4. Straddle payoff (8.1), option values reported at $S=100$, $t=0$. Note in this case, each timestep requires the solution of four one-dimensional linear PDEs.

American constraint handled explicitly.

one-dimensional PDE in a nonlinear iteration, then the piecewise constant policy method in Table 10 requires about twice the work of the implicit control method in Table 7.

From a practical point of view, the application of piecewise constant policies may be worrisome, since we typically need to compute the first and second derivatives (w.r.t. $S$ ) in financial applications. Imposing max, min constraints at the end of each timestep might be expected to cause non-smoothness in the solution, which is magnified by computing the derivatives. In Figure 3 we show the solution gamma $\left(V_{S S}\right.$ at $\left.\tau=T\right)$, for the borrow fee example, with American early exercise. We use the complete piecewise constant policy method here (including explicit American constraint), which is expected to be a worst case for computing $V_{S S}$. Figure 3 shows that gamma is certainly smooth enough for any practical hedging application.

As noted in the discussion after Remark 6.7, there exist examples of nonconvergence of policy type iteration schemes for HJBI equations. In our experience, we have never seen this occur for time dependent option pricing problems. However, we have seen very slow convergence.

Remark 8.1 (Choice of Scheme) Although we have not seen seen cases where the policy type iteration schemes fail for HJBI equations in option pricing, it is likely prudent to use a piecewise constant policy approximation scheme for at least some of the controls, so that the implicit control problem reduces to a sup or inf control. In this way, we are guaranteed convergence of the iteration method.

However, we still may seek to use the piecewise constant policy method to further reduce the number of controls solved implicitly. Solution of the local control problem may be quite difficult, as in the example of a controlled PIDE, which would arise when modelling jump processes.

We have shown that it is straightforward to approximate some of the controls by piecewise constants, and other controls can be solved implicitly. Since the piecewise constant approximation generally requires solution of an additional linear PDE for each discretized control, it is advantageous to make a judicious choice for which controls should use the piecewise constant approximation. In particular, it may be advantageous to use an implicit control approach for continuous controls, and a piecewise constant approach for discrete controls. 


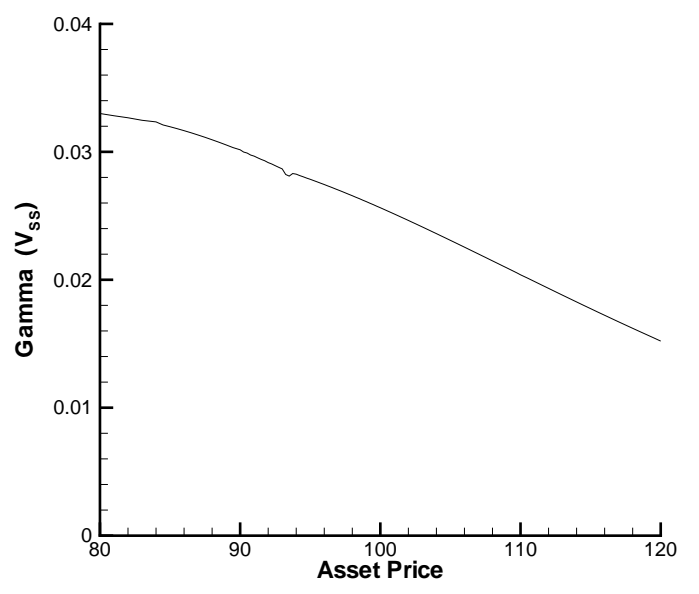

FiguRE 3: Gamma $\left(V_{S S}\right)$ for a straddle. Stock borrowing fee example as described in Section 2.2, long position with American early exercise. Input parameters are given in Table 4. Explicit evaluation of American constraint and piecewise constant policy timestepping (Section 7).

\section{Conclusion}

In this paper, we have studied the solution of optimal control problems in option pricing. We discretize the control problem directly, and do not to attempt to simplify the problem by analytically determining the optimal control. It turns out to be straightforward to analyze the discrete control problem in order to ensure that the discretization method is consistent, stable and monotone, and hence guarantee convergence to the viscosity solution. The control formulation also allows us to use a Newton-like iteration scheme to solve the implicit control discretized equations. For the HJB equation, global convergence of the policy (Newton-type) iteration is guaranteed.

In some cases, it may be difficult to solve the local control problems at each node. As well, in the case of HJBI equations, convergence of the policy type iteration scheme is not ensured. Although we can use a relaxation method in this situation, convergence is typically slow. In these cases, it may be advantageous to use a piecewise constant policy approximation. This reduces a complex nonlinear PDE to a set of linear PDEs at each timestep.

We note that all the proofs and methods described above can be trivially extended to higher dimensional problems, provided that we can discretize the operator

$$
\sum_{i=1}^{n_{\operatorname{dim}}} \sum_{j=1}^{n_{\operatorname{dim}}} a_{i, j} \frac{\partial^{2}}{\partial x_{i} x_{j}}
$$

so that the discrete equations yield a negative $M$ matrix. In some special cases [33], it is possible to use a specific grid spacing which ensures that the discrete equations give rise to an $M$ matrix. In other cases, it may be possible to rotate the grid so as to eliminate the cross-derivative term. In general, however, the situation is not satisfactory, and this is the subject of ongoing research. In any case, the major difficulty reduces to the classical problem of constructing a positive coefficient 
discretization of operator (9.1) [53].

In the future, we plan to investigate cases where the when the underlying process is a jump process, which would result in a controlled partial integro differential equation (PIDE) rather than a PDE.

\section{Appendices}

\section{A Derivation of the Nonlinear PDEs}

In this appendix, we give a brief derivation of the nonlinear option pricing PDEs for the cases of unequal borrowing and lending rates, as well as the case with stock borrowing fees.

\section{A.1 Unequal Borrowing and Lending Rates}

Consider a short position in the contingent claim with value $V(S, t)$. Consider the portfolio

$$
\Pi=-V+\alpha S
$$

where $\alpha$ is number of shares held long. The above portfolio is augmented (as usual) with the bank account $B$, so that at any instant

$$
\Pi_{T}=\Pi+B=0
$$

so that $B=V-\alpha S$. If $\Pi_{T}$ is riskless, then

$$
d \Pi_{T}=d \Pi+d B=d \Pi+\rho(B) B d t=0,
$$

where $\rho(x)$ is defined in equation (2.8). Note that we have included the effect of different borrowing and lending rates in equation (A.3), since the rate earned/paid on the bank account will depend on the sign of $B$.

Assuming the process (2.1), eliminating risk by setting $\alpha=V_{S}$, and using Ito's Lemma, then for a short position we obtain (from equations (A.3))

$$
V_{t}+\frac{\sigma^{2} S^{2}}{2} V_{S S}+\rho\left(V-S V_{S}\right)\left(S V_{S}-V\right)=0
$$

A similar argument for the long case gives the result in equation (2.7).

\section{A.2 Stock Borrowing Fees}

Although a fairly complex sequence of transactions takes place when stock is borrowed in order to go short [21], the end result is that the holder of the short position will not receive the rate $r_{l}$ on the proceeds of the short sale, but rather effectively receives $r_{l}-r_{f}$, where $r_{f}$ is the borrow fee. Typically, $r_{f}$ can be about 40 bps (.4\%) [52].

Consider a short position in the claim, and define $\Pi_{T}, \Pi, B$ as in Appendix A.1. If $\Pi_{T}$ is riskless, then we have

$$
d \Pi+d B=0 .
$$


Now, we have to distinguish between the two cases: $\alpha=V_{S}>0$, and $\alpha=V_{S}<0$. If $V_{S}<0$ (a short position in the underlying), we have

$$
d B=\left(\rho(V) V-\left(r_{l}-r_{f}\right) S V_{S}\right) d t,
$$

where $\rho(x)$ is defined in equation (2.8). On the other hand, if $V_{S}>0$ (a long position in the underlying), then

$$
d B=\rho\left(V-S V_{S}\right)\left(V-S V_{S}\right) d t .
$$

Assuming the process (2.1), eliminating risk by setting $\alpha=V_{S}$, and using Ito's Lemma, then from equations (A.5-A.7) we obtain for a short position

$$
V_{t}+\frac{\sigma^{2} S^{2}}{2} V_{S S}+H\left(V_{S}\right)\left[\rho\left(V-S V_{S}\right)\left(S V_{S}-V\right)\right]+H\left(-V_{S}\right)\left[\left(r_{l}-r_{f}\right) S V_{S}-\rho(V) V\right]=0,
$$

with $H(x)$ defined in equation (2.11). A similar argument for the long case gives the result in equation (2.10).

\section{B Some Useful Properties}

We gather in this Appendix some convenient properties which we will reference in the main text. Suppose $X(x), Y(x)$ are functions defined for some set of points $x \in D_{1}$. Then

$$
\begin{aligned}
& \sup _{x} X(x)-\sup _{y} Y(y) \leq \sup _{x}(X(x)-Y(x)), \\
& \inf _{x} X(x)-\inf _{y} Y(y) \geq \inf _{x}(X(x)-Y(x)),
\end{aligned}
$$

from which we can deduce

$$
\begin{aligned}
& \inf _{x}(X(x)-Y(x)) \leq \sup _{x} X(x)-\sup _{y} Y(y) \leq \sup _{x}(X(x)-Y(x)) \\
& \inf _{x}(X(x)-Y(x)) \leq \inf _{x} X(x)-\inf _{y} Y(y) \leq \sup _{x}(X(x)-Y(x))
\end{aligned}
$$

and

$$
\begin{aligned}
&\left|\sup _{x} X(x)-\sup _{y} Y(y)\right| \leq \sup _{x} \mid(X(x)-Y(x) \mid \\
&\left|\inf _{x} X(x)-\inf _{y} Y(y)\right| \leq \sup _{x}|X(x)-Y(x)| .
\end{aligned}
$$

Also from the above that, if $X(x, y), Y(x, y)$ are functions defined for the points $(x, y) \in D_{2}$, then

$$
\begin{aligned}
& \inf _{x} \inf _{y}(X(x, y)-Y(x, y)) \leq \inf _{x} \sup _{y} X(x, y)-\inf _{w} \sup _{z} Y(w, z) \leq \sup _{x} \sup _{y}(X(x, y)-Y(x, y)) \\
& \left|\inf _{x} \sup _{y} X(x, y)-\inf _{w} \sup _{z} Y(w, z)\right| \leq \sup _{x} \sup _{y}|X(x, y)-Y(x, y)|
\end{aligned}
$$

which also hold if the inf sup is replaced by sup inf in equations (B.6-B.7). 


\section{Discrete Equation Coefficients}

Let $Q_{i}^{n}$ denote the vector of optimal controls at node $i$, time level $n$ and set

$$
a_{i}^{n+1}=a\left(S_{i}, \tau^{n}, Q_{i}^{n}\right), b_{i}^{n+1}=b\left(S_{i}, \tau^{n}, Q_{i}^{n}\right), \quad c_{i}^{n+1}=c\left(S_{i}, \tau^{n}, Q_{i}^{n}\right) .
$$

Then, we can use central, forward or backward differencing at any node.

Central Differencing:

$$
\begin{aligned}
\alpha_{i, \text { central }}^{n} & =\left[\frac{2 a_{i}^{n}}{\left(S_{i}-S_{i-1}\right)\left(S_{i+1}-S_{i-1}\right)}-\frac{b_{i}^{n}}{S_{i+1}-S_{i-1}}\right] \\
\beta_{i, \text { central }}^{n} & =\left[\frac{2 a_{i}^{n}}{\left(S_{i+1}-S_{i}\right)\left(S_{i+1}-S_{i-1}\right)}+\frac{b_{i}^{n}}{S_{i+1}-S_{i-1}}\right] .
\end{aligned}
$$

Forward/backward Differencing: $\left(b_{i}^{n}>0 / b_{i}^{n}<0\right)$

$$
\begin{aligned}
\alpha_{i, \text { forward/backward }}^{n} & =\left[\frac{2 a_{i}^{n}}{\left(S_{i}-S_{i-1}\right)\left(S_{i+1}-S_{i-1}\right)}+\max \left(0, \frac{-b_{i}^{n}}{S_{i}-S_{i-1}}\right)\right] \\
\beta_{i, \text { forward/backward }}^{n} & =\left[\frac{2 a_{i}^{n}}{\left(S_{i+1}-S_{i}\right)\left(S_{i+1}-S_{i-1}\right)}+\max \left(0, \frac{b_{i}^{n}}{S_{i+1}-S_{i}}\right)\right] .
\end{aligned}
$$

\section{References}

[1] A. L. Amadori. Differential and Integro-differential Nonlinear Equations of Degenerate Parabolic Type Arising in the Pricing of Derivatives in Incomplete Markets. PhD thesis, University of Rome, La Sapienza, 2001.

[2] A. L. Amadori. Nonlinear integro-differential evolution problems arising in option pricing: A viscosity solution approach. Journal of Differential and Integral Equations, 16:787-811, 2003.

[3] L. Andersen, J. Andreasen, and R. Brotherton-Ratcliffe. The passport option. Journal of Computational Finance, 1(3):15-36, Spring 1998.

[4] M. Avellaneda, A. Levy, and A. Parás. Pricing and hedging derivative securities in markets with uncertain volatilities. Applied Mathematical Finance, 2:73-88, 1995.

[5] G. Barles. Convergence of numerical schemes for degenerate parabolic equations arising in finance. In L. C. G. Rogers and D. Talay, editors, Numerical Methods in Finance, pages 1-21. Cambridge University Press, Cambridge, 1997.

[6] G. Barles and J. Burdeau. The Dirichlet problem for semilinear second-order degenerate elliptic equations and applications to stochastic exit time control problems. Communications in Partial Differential Equations, 20:129-178, 1995. 
[7] G. Barles, CH. Daher, and M. Romano. Convergence of numerical shemes for parabolic eqations arising in finance theory. Mathematical Models and Methods in Applied Sciences, 5:125-143, 1995.

[8] G. Barles and E. Jakobsen. Error bounds for monotone approximation schemes for parabolic Hamilton-Jacobi-Bellman equations. Mathematics of Computation, 76:1861-1893, 2007.

[9] G. Barles and E. Rouy. A strong comparison result for the Bellman equation arising in stochastic exit time control problems and applications. Communications in Partial Differential Equations, 23:1995-2033, 1998.

[10] G. Barles and P.E. Souganidis. Convergence of approximation schemes for fully nonlinear equations. Asymptotic Analysis, 4:271-283, 1991.

[11] E. Bayraktar and V. Young. Pricing options in incomplete markets via the instantaneous Sharpe Ratio. Working paper, University of Michigan, 2005.

[12] F. E. Benth, K.H. Karlsen, and K. Reikvam. Optimal portfolio management management rules in a nonguaussian market with durability and intertemporal substitution. Finance and Stochastics, 5:447-467, 2001.

[13] Y. Bergman. Option pricing with differential interest rates. Review of Financial Studies, 8:475-500, 1995.

[14] M. Breton and P. L'Ecuyer. Approximate solutions to continuous stochastic games. In R. Hamamainen and H. Ehtamo, editors, Differential Games: Developments in Modelling and Computation, pages 258-264. Springer Verlag, New York, 1991. Lecture Notes in Control and Information Sciences, Vol. 156.

[15] J. Ph. Chancellor, B. Oksendal, and A. Sulem. Combined stochastic control and optimal stopping and application to numerical approximation of combined stochastic and impulse control. Stochastic Financial Mathematics, 237:149-173, 2002. Proceedings of the Steklov Institute, Moscow, Editor: A.N. Shiryaev.

[16] S. Chaumont. A strong comparison result for viscosity solutions to Hamilton-Jacobi-Bellman equations with Dirichlet conditions on a non-smooth boundary. Working paper, Institute Elie Cartan, Université Nancy I, 2004.

[17] R. Cont and P. Tankov. Financial Modelling with Jump Processes. Chapman \& Hall/CRC, 2004.

[18] M. G. Crandall, H. Ishii, and P. L. Lions. User's guide to viscosity solutions of second order partial differential equations. Bulletin of the American Mathematical Society, 27:1-67, 1992.

[19] M. Dai, Y.K. Kwok, and J. Zong. Guaranteed minimum withdrawal benefit variable annuities. To appear, Mathematical Finance, 2007.

[20] Y. d'Halluin, P.A. Forsyth, and K.R. Vetzal. Robust numerical methods for contingent claims under jump diffusion processes. IMA Journal of Numerical Analysis, 25:87-112, 2005. 
[21] D. Duffie, N. Garleanu, and L. H. Pedersen. Securities lending, shorting and pricing. Journal of Financial Economics, 66:307-339, 2002.

[22] P.A. Forsyth and K.R. Vetzal. Quadratic convergence of a penalty method for valuing American options. SIAM Journal on Scientific Computation, 23:2096-2123, 2002.

[23] S.L. Heston, M. Lowenstein, and G.A. Willard. Options and bubbles. Review of Financial Studies, 20:359-390, 2007.

[24] E.R. Jakobsen, K.H. Karlsen, and C. La Chioma. Error Estimates for Approximate Solutions to the Bellman Equations Associated with Controlled Jump Diffusions . Working paper, Center for Mathematics and its Applications, University of Oslo , 2005.

[25] N.V. Krylov. Approximating value functions for controlled degenerate diffusion processes by using piece-wise constant policies. Electronic Journal of Probability, 4(2):1-19, 1999.

[26] N.V. Krylov. On the rate of convergence of finite difference approximations for Bellman's equations with variable coefficients. Probability Theory and Related Fields, 117:1-16, 2000.

[27] H.J. Kushner and P.G Dupuis. Numerical Methods for Stochastic Control Problems in Continuous Time. Springer-Verlag, New York, 1991.

[28] H. E. Leland. Option pricing and replication with transaction costs. Journal of Finance, 40:1283-1301, 1985.

[29] F. Da Lio and O. Ley. Uniquness results for second order Bellman-Isaacs equations under quadratic growth assumptions and applications. Working paper, University of Torino, 2004.

[30] T. Lyons. Uncertain volatility and the risk free synthesis of derivatives. Applied Mathematical Finance, 2:117-133, 1995.

[31] M. Milevsky, S. Promislow, and V. Young. Financial valuation of mortality risk via the instantaneous Sharpe ratio. Working paper, York University, 2005.

[32] M. Mnif and A. Sulem. Optimal risk control under excess of loss reinsurance. Working paper, Université Paris 6, 2001.

[33] B Oksendal and A. Sulem. Applied Stochastic Control of Jump Diffusions. Springer Verlag, Berlin, 2005.

[34] O.A. Oleinik and E.V. Radkevic. Second Order Equations with Nonnegative Characteristic Form. American Mathematical Society, Providence, 1973.

[35] H. Pham. On some recent aspects of stochastic control and their applications. Probability Surveys, 2:506-549, 2005.

[36] M. Pollatschek and B. Avi-Itzhak. Algoritms for stochastic games with geometrical interpretation. Management Science, 15:399-415, 1969. 
[37] D. M. Pooley. Numerical Methods for Nonlinear Equations in Option Pricing. PhD thesis, School of Computer Science, University of Waterloo, 2003.

[38] D.M. Pooley, P.A. Forsyth, and K.R. Vetzal. Numerical convergence properties of option pricing PDEs with uncertain volatility. IMA Journal of Numerical Analysis, 23:241-267, 2003.

[39] M. Puterman. Markov Decison Processes: Discrete Stochastic Dynamic Programming. Wiley, New York, 1994.

[40] M. Puterman and S. Brumelle. On the convergence of policy iteration in stationary dynamic programming. Mathematics of Operations Research, 4:60-69, 1979.

[41] R. Rannacher. Finite element solution of diffusion problems with irregular data. Numerische Mathematik, 43:309-327, 1984.

[42] M. Santos and J. Rust. Convergence properties of policy iteration. SIAM Journal on Control and Optimization, 42:2094-2115, 2004.

[43] S. Shreve and J. Vecer. Options on a traded account: vacation calls, vacation puts, and passport options. Finance and Stochastics, 4:255-274, Spring 2000.

[44] P.E. Souganidis. Approximation schemes for viscosity solutions of Hamilton-Jacobi equations. Journal of Differential Equations, 59:1-43, 1985.

[45] B. Tolwinski. Newton type methods for stochastic games. In T. Basar and P. Bernhard, editors, Differential Games and Applications, pages 128-144. Springer Verlag, New York, 1989. Lecture Notes in Control and Information Sciences, Vol. 119.

[46] B. Tolwinski. Solving dynamic games via Markov game approximations. In R. Hamamainen and H. Ehtamo, editors, Differential Games: Developments in Modelling and Computation, pages 265-274. Springer Verlag, New York, 1991. Lecture Notes in Control and Information Sciences, Vol. 156.

[47] R. S. Varga. Matrix Iterative Analysis. Springer Verlag, 2000.

[48] J. Van Der Wal. Discounted Markov games: Generalized policy iteration method. Journal of Optimization Theory and Applications, 25:125-138, 1978.

[49] J. Wang and P.A. Forsyth. Maximal use of central differencing for Hamilton-Jacobi-Bellman PDEs in finance. To appear, SIAM Journal on Numerical Analysis, 2007.

[50] H. Windcliff, P.A. Forsyth, and K.R. Vetzal. Analysis of the stability of the linear boundary condition for the Black-Scholes equation. Journal of Computational Finance, 8(Fall), 2004.

[51] H. Windcliff, J. Wang, P.A. Forsyth, and K.R. Vetzal. Hedging with a correlated asset: Solution of a nonlinear pricing PDE. Journal of Computational and Applied Mathematics, 200:86-115, 2007.

[52] R. Zvan. Bear-Stearns, private communication. 2005. 
[53] R. Zvan, P.A. Forsyth, and K.R. Vetzal. Negative coefficients for two factor option pricing models. Journal of Computational Finance, 7 (Fall):37-73, 2003. 